\title{
ISL1 cardiovascular progenitor cells for cardiac repair after myocardial infarction
}

\author{
Oscar Bartulos, ${ }^{1,2}$ Zhen Wu Zhuang, ${ }^{1}$ Yan Huang, ${ }^{1}$ Nicole Mikush, ${ }^{1}$ Carol Suh,, ${ }^{1,2}$ Alda Bregasi, ${ }^{1}$ \\ Lin Wang, ${ }^{2,3}$ William Chang, ${ }^{4}$ Diane S. Krause, ${ }^{2,3,5,6}$ Lawrence H. Young, ${ }^{1,7}$ Jordan S. Pober, ${ }^{6,8,9}$ \\ Yibing Qyang, 1,2,6,9 \\ ${ }^{1} Y a l e$ Cardiovascular Research Center, Section of Cardiovascular Medicine, Department of Internal Medicine, ${ }^{2}$ Yale Stem \\ Cell Center, ${ }^{3}$ Department of Laboratory Medicine, ${ }^{4}$ Department of Internal Medicine, Section of Nephrology, ${ }^{5}$ Department \\ of Cell Biology, ${ }^{6}$ Pathology, ${ }^{7}$ Cellular and Molecular Physiology, ${ }^{8} 1$ mmunobiology, and ${ }^{9}$ Vascular Biology and Therapeutics \\ Program, Yale University, New Haven, Connecticut, USA.
}

\begin{abstract}
Cardiovascular progenitor cells (CPCs) expressing the ISL1-LIM-homeodomain transcription factor contribute developmentally to cardiomyocytes in all 4 chambers of the heart. Here, we show that ISL1-CPCs can be applied to myocardial regeneration following injury. We used a rapid 3D methylcellulose approach to form murine and human ISL1-CPC spheroids that engrafted after myocardial infarction in murine hearts, where they differentiated into cardiomyocytes and endothelial cells, integrating into the myocardium and forming new blood vessels. ISL1CPC spheroid-treated mice exhibited reduced infarct area and increased blood vessel formation compared with control animals. Moreover, left ventricular (LV) contractile function was significantly better in mice transplanted with ISL1-CPCs 4 weeks after injury than that in control animals. These results provide proof-of-concept of a cardiac repair strategy employing ISL1-CPCs that, based on our previous lineage-tracing studies, are committed to forming heart tissue, in combination with a robust methylcellulose spheroid-based delivery approach.
\end{abstract}

Conflict of interest: The authors declare that no conflict of interest exists.

Submitted: January 12, 2015

Accepted: May 19, 2016

Published: July 7, 2016

Reference information: JCI Insight. 2016;1(10):e80920. doi:10.1172/jci.insight.80920.

\section{Introduction}

Heart failure caused by myocardial infarction (MI; death of heart muscle) is a leading cause of morbidity and mortality worldwide. Cardiovascular progenitor cells (CPCs) represent a potentially valuable source of cells for cardiac regeneration after MI. While primary heart tissue-derived CPCs, such as those marked by c-Kit, Sca-1, or CD105 (cardiospheres), provide a possible approach to cell-based therapy, their derivation is restricted by limited tissue accessibility, and the therapeutic function of these cells may decline with age (1-5). Additionally, the contribution of these CPCs to the formation of cardiomyocytes appears to be minimal $(1,2)$. In contrast, CPCs derived from embryonic stem cells (ESCs) or induced pluripotent stem cells could represent a valuable alternative cell source for cardiac repair due to their unlimited availability. ESC-derived Flk- $1^{+} \mathrm{PdgfR}-\alpha^{+} \mathrm{CPCs}$ have been shown to enhance cardiac function of injured rodent hearts $(6,7)$. Additionally, SSEA- $1^{+} \mathrm{CPCs}$ derived from ESCs have been recently administered to a single patient with heart failure (8), suggesting a potential feasibility of these CPCs in a clinical setting. To understand the therapeutic potential of these ESC-derived CPCs, it would be important to fully investigate the biology of these cells, such as the engraftment efficiency after implantation, cell tracking by imaging analysis, and fate-mapping by lineage tracing studies.

Recent lineage tracing analyses reveal that Is11-expressing CPCs (Is11-CPCs) contribute to the major population of cardiomyocytes in all 4 chambers of the heart, as well as vascular smooth muscle cells (SMCs) and endothelial cells (ECs) (9-11). ESC-derived ISL1-CPCs generated by our group and others are the functional equivalent of their heart tissue-derived counterparts and have the potential to differentiate into all 3 cardiovascular lineages in the heart (11-13). Prior studies using ESC-derived CPCs based their identification and isolation on Cre-recombinase activity directed by the ISL1 promoter $(12,14)$. Such progenitors might also contain a fraction of extracardiac cells due to the expression of ISL1 in other tissues besides the heart (15).

To isolate ISL1-CPCs, we employed a murine ESC line in which GFP expression is directed by a fragment of the Mef2c gene that is specifically expressed within the ISL1 domain of the anterior heart field, thus enabling the derivation of mouse ISL1-CPCs (mISL1-CPCs) with authentic cardiac potential (13). Based on a human ESC (hESC) cardiomyocyte differentiation approach (16) with optimization, we have established 
a robust platform to derive highly enriched hISL1-CPCs that are doubly positive for CPC markers ISL1 and NKX2.5 (94\% ISL1 $\left.{ }^{+} / \mathrm{NKX} 2.5^{+}\right)$from ESCs.

A major hurdle faced in the use of CPCs for tissue repair has been stable engraftment. Previous studies have reported that implantation of cardiospheres formed on poly-D-lysine-coated dishes led to improved cell engraftment and cardiac function, but these cardiospheres contained cells with minimal cardiomyocyte differentiation and were laborious to derive (3-6 weeks) $(5,17)$. By applying a rapid (12- to 24-hour) methylcellulose-based approach (18), for the first time to our knowledge in cardiac repair, we generated ISL1CPC spheroids and examined their cardiovascular differentiation in vitro and in murine hearts after MI in vivo. In addition, we investigated the survival of these spheroids over time via luciferase-based live imaging and analyzed the effects of spheroids on cardiac remodeling and heart contractile function. Moreover, we explored whether ISL1-CPC spheroid-produced growth factors may protect cardiomyocytes under hypoxic conditions and/or reduce cardiac fibrosis. This is the first study of cardiac repair to our knowledge using ISL1-CPCs, an understudied cell type that has important potential for reparative therapy.

\section{Results}

Generation of a double-transgenic mESC line of ISL1-CPCS. We created a double-transgenic mESC line expressing GFP directed by a 449-bp anterior heart field enhancer contained in the Mef2c gene that is specifically expressed within the ISL1-CPC domain of the anterior heart field, as well as red fluorescent protein (RFP) and firefly luciferase expressed under the control of a ubiquitin promoter (Supplemental Figure 1; supplemental material available online with this article; doi:10.1172/jci.insight.80920DS1). This approach enabled purification of mISL1-CPCs with cardiac origin by GFP on embryoid day 5.5 (EB 5.5) and in vivo tracking by luciferase imaging after implantation.

Enhanced mISL1-CPC cardiac differentiation in methylcellulose-induced spheroids. Cells induced to form a 3D spherical structure have the potential to engraft and survive more effectively than a single cell suspension in the injured heart after implantation, but this approach required about 3-6 weeks (5). We thus sought to establish a rapid approach to generate mISL1-CPC spheroids for cardiac repair. We tested 3 different methods for spheroid formation: (i) hanging drops; (ii) forced aggregation in 96-well plates with V-shape bottoms (96-V plates); and (iii) methylcellulose-induced aggregation in low attachment in 96-well plates with U-shape bottoms (96-U plates). Starting with 1,000 cells per well or drop, spheroids were detected only in the presence of methylcellulose in the 96-U plates after 24 hours (Figure 1A). In contrast, mISL1-CPCs suspended in hanging drops could only aggregate into tiny cell clusters but failed to form spheroids (Figure 1A). Interestingly, mISL1-CPCs cultured in the 96-V plates after forced aggregation attached to the bottom of the wells without spheroid formation (Figure 1A). We observed that methylcellulose is required for spheroid formation, since in its absence, most of the mISL1-CPCs died and the surviving ones attached to the wells (Figure 1A).

Varying numbers of cells were tested to optimize the mISL1-CPC spheroid approach in methylcellulose. We ultimately chose to make spheroids with 1,000 cells, based on size and the time needed for spheroid formation. Twenty-four hours after mISL1-CPCs were seeded in the presence of methylcellulose, the vast majority of the cells typically clustered into spheroids with diameters ranging from 100-130 $\mu \mathrm{m}$ (Figure 1A). This size was thought to be suitable for intramyocardial injection into injured hearts.

Next, we examined the cardiac differentiation potential of mISL1-CPC spheroids in culture before implantation in vivo. As Matrigel enhances cell survival after implantation (19), we chose to use Matrigel as a cell carrier for cell transplantation. We plated mISL1-CPC spheroids on Matrigel-coated plates for in vitro cardiac differentiation to more closely mimic the in vivo differentiation environment. mISL1-CPCs were also plated as monolayer culture (mISL1-CPC single cells) for comparison of their cardiac differentiation potential. Smooth muscle $\alpha$-actin (SMA) is expressed in early-stage cardiomyocytes, as well as in SMCs (20). Although no significant differences were observed in the percentage of total SMA-positive cells (Figure 1D), mISL1-CPC spheroids showed a marked increase (3-fold) in the number of cardiomyocytes, which were double-positive for SMA and cardiac troponin T (cTnT), a specific marker of cardiomyocytes, compared with mISL1-CPC single cells ( $30 \pm 4 \%$ vs. $10 \pm 2 \%$ ) (Figure $1, \mathrm{~B}$ and D). Importantly, mISL1-CPC spheroids also showed striated sarcomeric structure, as well as synchronous beating activities (Figure 1C and Movies 1 and 2), suggesting the true cardiac potential of these CPCs.

We next analyzed the molecular signaling pathways responsible for mISL1-CPC spheroid formation, using small molecule inhibitor screening. ICG001 and TNP470 both disrupted the process (Supplemental Figure 2A). ICG001 interferes with canonical Wnt signaling by blocking the interaction between $\beta$-catenin 
A

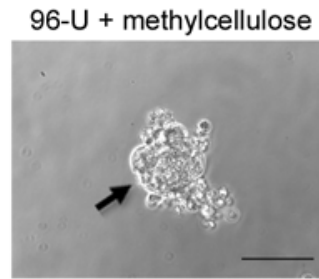

Hanging drops

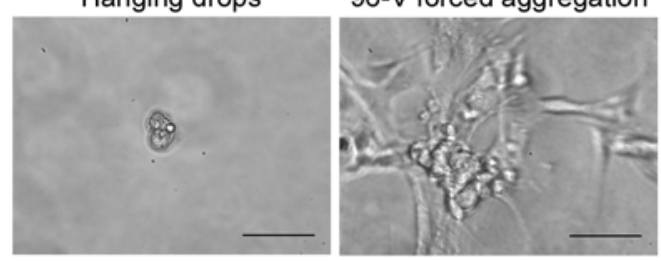

96- $\mathrm{V}$ forced aggregation

96-U - methylcellulose
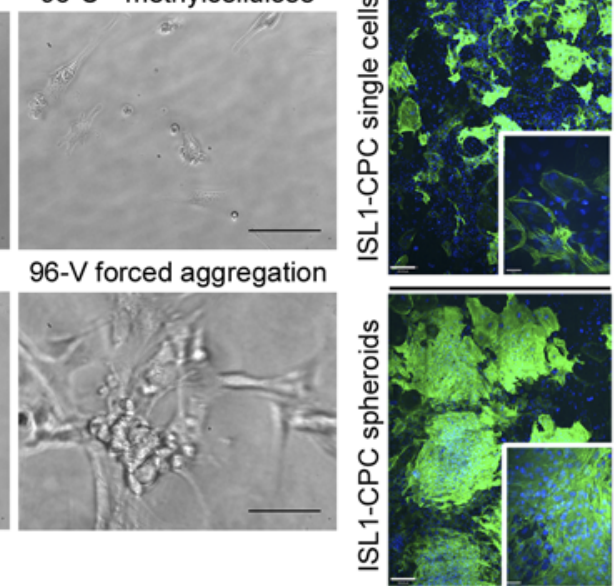

C

B cTnT Hoechst

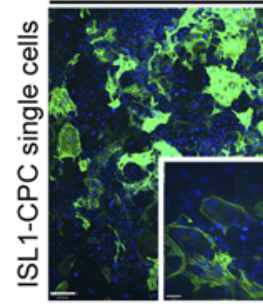

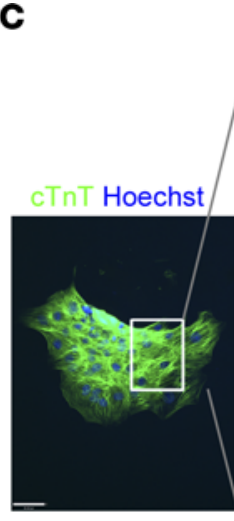

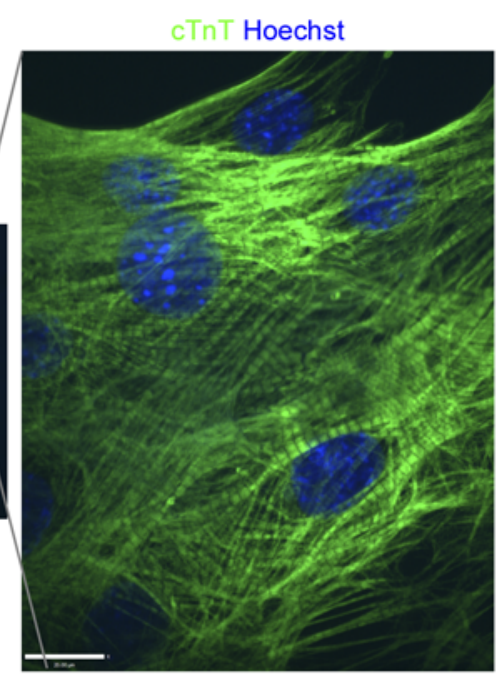

D

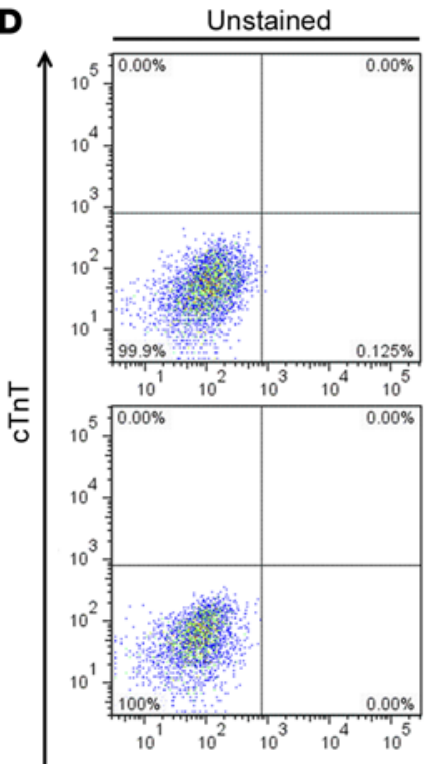

IgGs Control
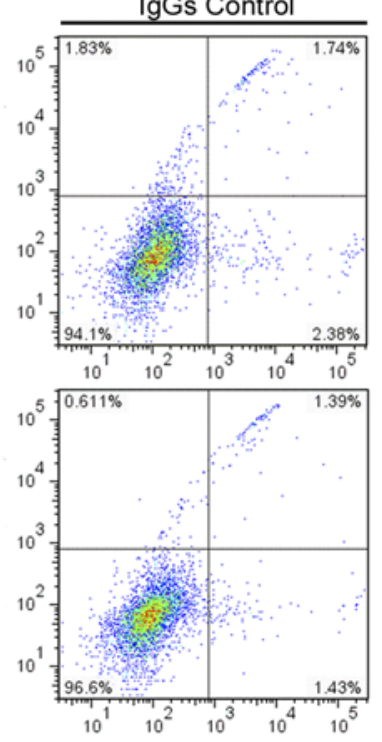
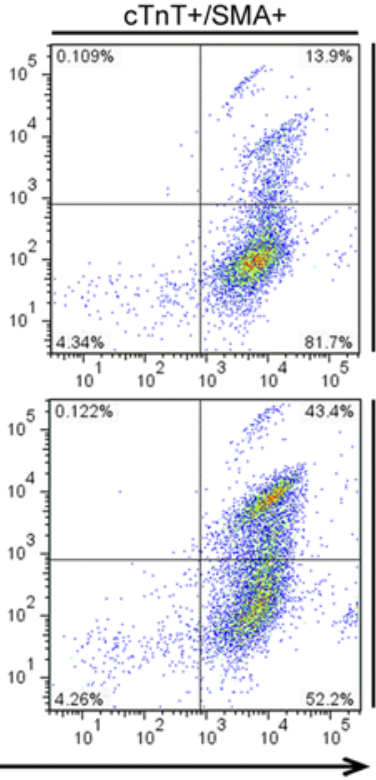

SMA+

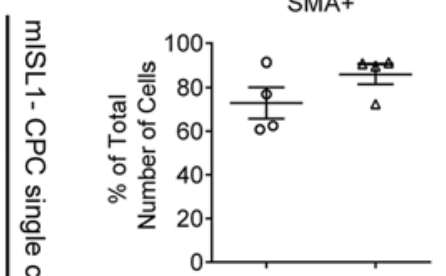

- mISL1-CPC single cells $\triangle$ mISL1-CPC spheroids

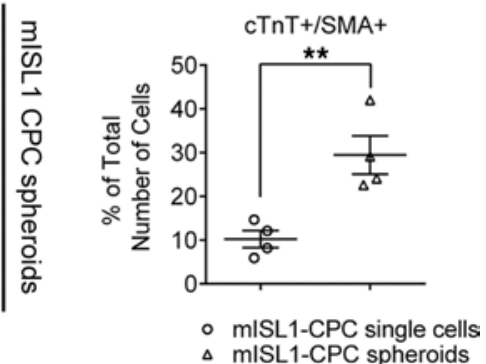

SMA

Figure 1. Cardiac differentiation of mouse ISL1 cardiovascular progenitor cell-derived spheroids (mISL1-CPC spheroids). (A) Test for spheroid formation using 96-U wells with (top left) or without (top right) methylcellulose, hanging drops (bottom left), or forced aggregation in 96-V wells (bottom right). Mouse ISL1-CPC spheroids were only formed in 96-U wells, in the presence of methylcellulose (top left, black arrow). Scale bars: 100 $\mu \mathrm{m}$. (B) Mouse ISL1CPCs were isolated by FACS and plated as monolayer culture (single cells) or spheroids on Matrigel-coated plates for 1 week. Cells were fixed and stained for cardiac troponin T (cTnT; green). Scale bar: $200 \mu \mathrm{m}$. Scale bar for inset pictures: $50 \mu \mathrm{m}$. (C) Staining for cTnT (green) in ISL1-CPC spheroids plated on the Matrigel-coated 4-well chamber slide for 6 days. Pictures represent $\times 20$ magnifications (Scale bar: $50 \mu \mathrm{m}$ ) on the left and $\times 90$ magnification (Scale bar: 20 $\mu \mathrm{m})$ on the right. Note in the $\times 90$ picture the presence of sarcomeric structures. (D) One week after plating on Matrigel, mISL1-CPC single cells or spheroids were subjected to FACS analysis to quantify the percentage of cardiomyocytes marked by the specific cardiac marker cTnT and the early cardiomyocyte marker smooth muscle $\alpha$-actin (SMA). Quantifications of SMA+ cells and cardiomyocytes (cTnT+/SMA+) from $n=4$ independent experiments (unpaired 2-tailed Student's $t$ test). ${ }^{* *} P<0.01$. See also Supplemental Figures 1 and 2 and Movies 1 and 2.

and CREB binding protein (CBP) (21), while TNP470 inhibits the noncanonical planar cell polarity pathway (22). To test whether ICG001 and TNP470 interfered with mISL1-CPC spheroid formation by inducing cell death, we next plated ISL1-CPCs on fibronectin-coated plates and treated them with these inhibitors for 24 hours. Vital staining revealed that ICG001 induced cell death, while no significant differences were observed between TNP470 and control (DMSO) treatments (Supplemental Figure 2A), suggesting that a noncanonical planar cell polarity pathway may play an essential role in spheroid formation. On the other hand, during the small molecule screening, we identified the ROCK kinase inhibitor Y27632 as an enhancer of mISL1-CPC spheroid formation. In the presence of Y27632, the time required to form the spheroids was reduced from 24 to 12 hours (Supplemental Figure 2B). 
A

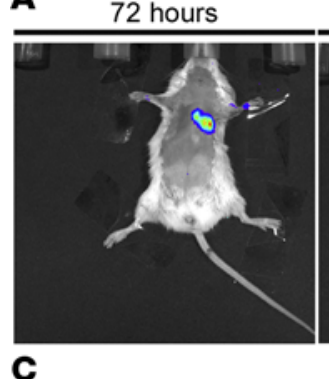

2 weeks

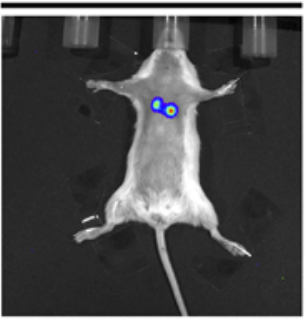

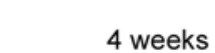

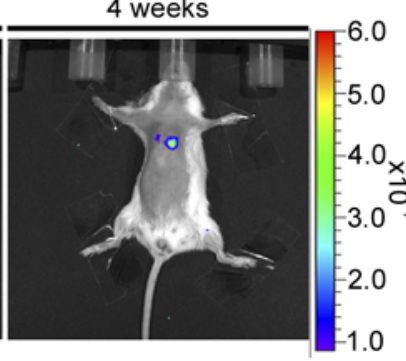

Luciferase In vivo bioluminescence quantification mouse ISL1-CPC spheroids

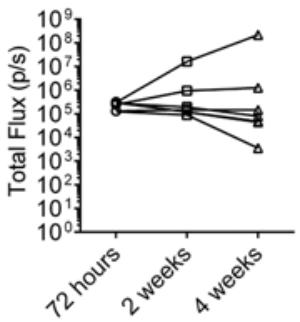

B 4 weeks Luciferase

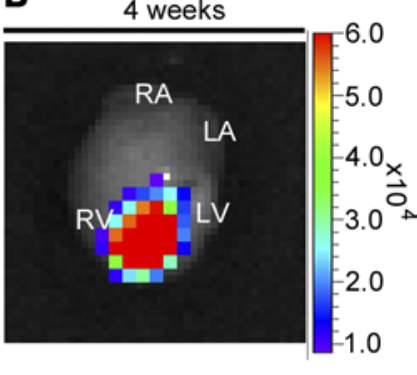

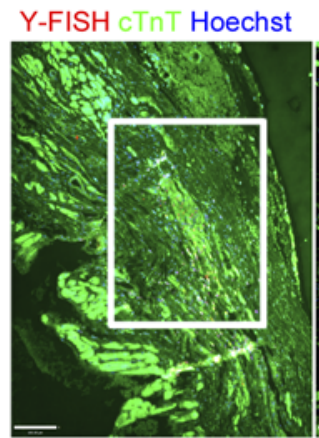

D

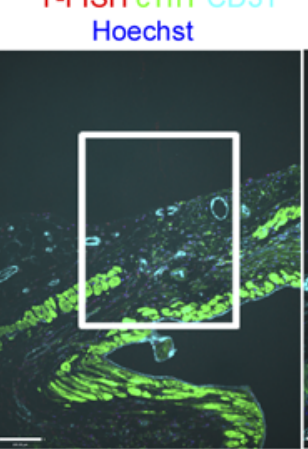

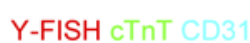
Hoechst

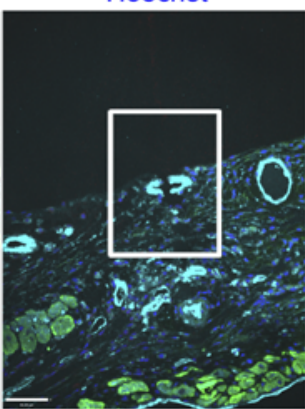

Y-FISH Hoechst

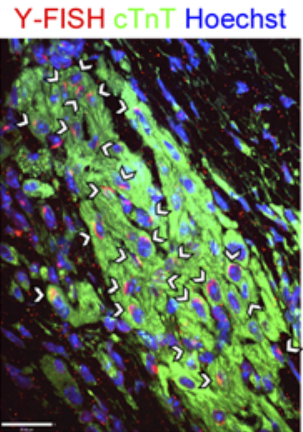

\begin{abstract}
Y-FISH Hoechst
\end{abstract}

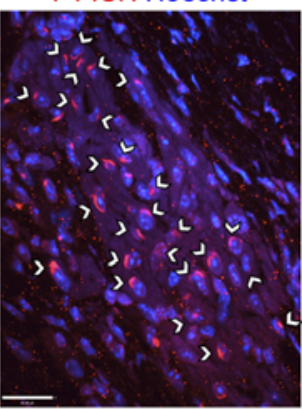
Y-FISH CD31 Hoechst Y-FISH Hoechst
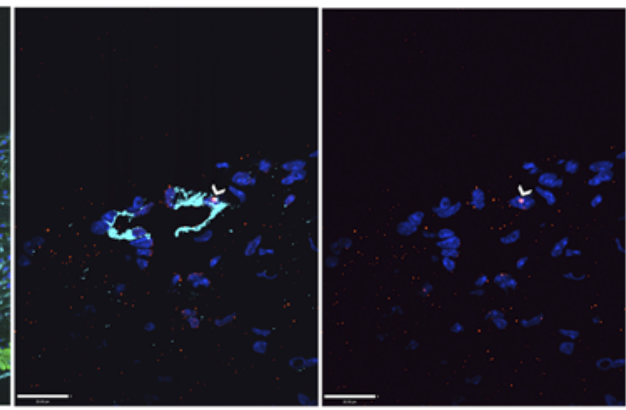

E

Y-chromosome positive cells

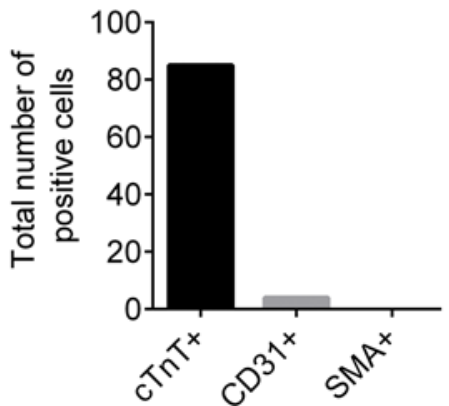

Figure 2. Mouse ISL1-CPC spheroid-derived cells in infarcted hearts. (A) Bioluminescent images in a representative live animal injected with mISL1-CPC spheroids and recorded at 3 different time points: left ( 72 hours); middle ( 2 weeks); right ( 4 weeks). The far right: Graph representing the bioluminescence signal quantified in live animals injected with mISL1-CPC spheroids, $n=7$. Statistical analysis performed with 1-way repeated measurements ANOVA with Greenhouse-Geisser correction $(P=0.35)$. (B) Bioluminescent image of a heart explanted 4 weeks after artery ligation and injection with mISL1-CPC spheroids. LA, left atrium; LV, left ventricle; RA, right atrium; RV, right ventricle. In $\mathbf{A}$ and $\mathbf{B}$, the numbers in the scale bars were introduced manually to enlarge the font size. (C and $\mathbf{D})$ Histological sections of explanted hearts 4 weeks after surgery, showing that mISL1-CPC spheroids differentiated into cardiomyocytes (C) and endothelial cells (D). Note the integration of donor endothelial cells in host blood vessels. Pictures in $\mathbf{C}$ represent $\times 10$ (left), $\times 20$ (second and third from left), and $\times 60$ (fourth and fifth from left) magnification. Pictures in D represent $\times 10$ (left), $\times 20$ (second from left), and $\times 60$ (third and fourth from left) magnification. White rectangles in $\times 10$ and $\times 20$ pictures represent the area magnified in $\times 20$ and $\times 60$, respectively. Y-chromosome

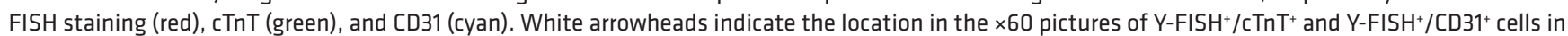
C and D, respectively. Scale bars: $100 \mu \mathrm{m}$ in $\times 10,50 \mu \mathrm{m}$ in $\times 20$, and $20 \mu \mathrm{m}$ in $\times 60$ pictures. (E) Quantification of double-positive Y-FISH/cTnT, Y-FISH/CD31, and Y-FISH/SMA cells in 4 infarcted hearts 4 weeks after surgery/cell injection. Seven slides, containing coronal sections, were analyzed per heart in the anatomical region where the bioluminescence was detected. See also Supplemental Figures 3 and 4.

mISL1-CPC spheroids engraft, survive, and differentiate into cardiomyocytes and ECs within infarcted hearts. We first compared the survival potential of mISL1-CPC single cells versus mISL1-CPC spheroids in vivo, after hindlimb injection in severe combined immunodeficiency-beige (SCID-bg) mice. Cell survival was assessed using bioluminescence imaging. Images were recorded 10 minutes and 24 hours after cell injection (Supplemental Figure 3A). While cells injected as spheroids were present, mISL1-CPCs injected as a single-cell suspension were undetectable 24 hours after implantation (Supplemental Figure 3A). Since mISL1-CPC spheroids had a markedly enhanced cardiomyocyte differentiation potential in culture (Figure 1) and better survival in vivo (Supplemental Figure 3A) compared with single cells, we selected the spheroid approach to test the functional benefits of mISL1-CPCs in infarcted hearts. 
A

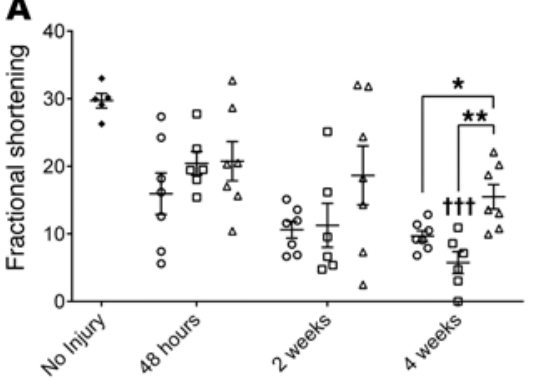

B
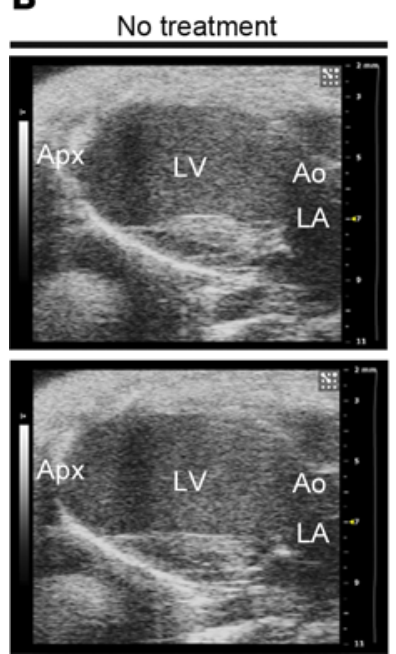
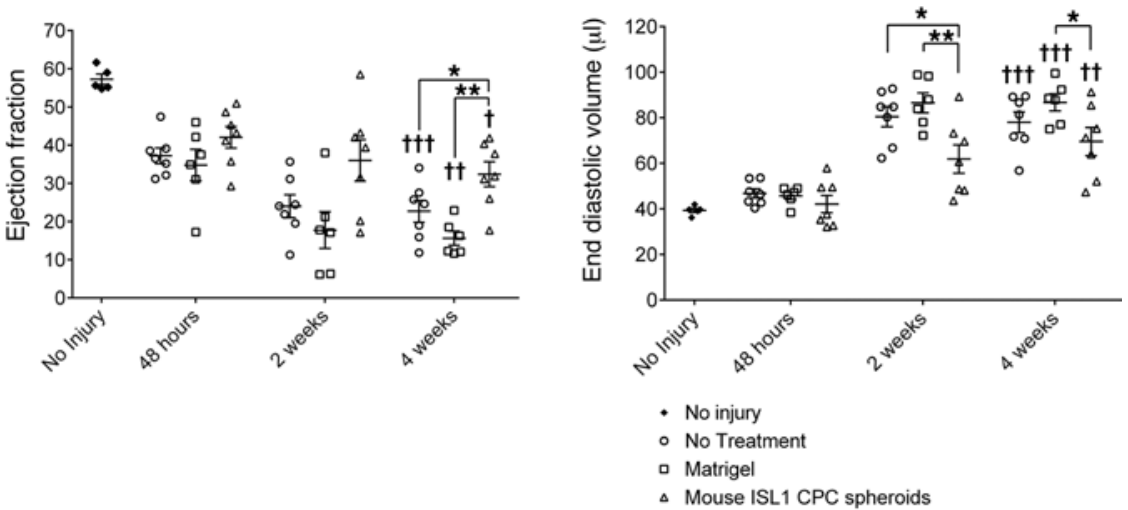

Figure 3. Left ventricular heart function in infarcted mice implanted with murine ISL1-CPC spheroids. (A) Fractional shortening (M-Mode) measured along the mid-left ventricular short axis, and ejection fraction (B-Mode) and end diastolic volume along the mid-part of parasternal long axis of mice subjected to ligation of the left coronary artery. No injury $(n=5)$, no treatment ( $n=7$ mice), Matrigel injection ( $n=6$ mice), mISL1-CPC spheroid injection ( $n=7$ mice). Three measurements were averaged per animal for each time point to obtain the M-Mode data. Samples were analyzed by 2 -way ANOVA and pair comparisons between groups using unpaired 2-tailed Student's $t$ test ( ${ }^{*} P<0.05$; ${ }^{*} P<0.01$ ), as well as pair comparisons for time points (48 hours vs. 4 weeks) within the same group using paired 2-tailed Student's $t$ test $\left({ }^{\dagger} P<0.05 ;{ }^{\dagger+} P \leq 0.01\right.$; $\left.{ }^{+\dagger} P<0.001\right)$. (B) Representative ultrasound images in systole and diastole recorded along the mid-left ventricular parasternal long axis (B-Mode) 4 weeks after surgery. Ao, aorta; Apx, apex; LA, left atrium; LV, left ventricle.See also Supplemental Figure 5, A and C.

MI was induced by permanent surgical ligation of the left coronary artery, and animals were randomly divided into 3 experimental groups: (i) ligation and no treatment; (ii) ligation and Matrigel injection (vehicle solution); and (iii) ligation and mISL1-CPC spheroid injection (500 spheroids containing 1,000 cells per spheroid). Bioluminescence imaging was performed to track cell survival and anatomical location in the mice 72 hours, 2 weeks, and 4 weeks after cell injection (Figure 2, A and B and Supplemental Figure $3, \mathrm{~B}$ and C). mISL1-CPC spheroid-derived cells were readily detected in live animals at all 3 time points, as well as in the explanted hearts 4 weeks after cell delivery (Figure 2, A and B and Supplemental Figure $3 C$ ). Other explanted organs (lungs, kidneys, spleen, and liver) were also evaluated for the presence of implanted cells. We detected bioluminescence signals primarily in the hearts of the injected animals (Figure $2 \mathrm{~B}$ ) with minimal presence in their lungs (data not shown). No other organs showed detectable signals of mISL1-CPC spheroid-derived cells. Importantly, no significant decrement (one-way repeated measurements ANOVA, $P=0.35$ ) in the magnitude of the bioluminescence signals was observed during the course of 4 weeks (Figure 2A and Supplemental Figure 3C), suggesting that mISL1-CPCs implanted as spheroids survive efficiently in the harsh environment of an infarcted heart. In addition, no teratomas were detected in any of the hearts analyzed.

We also assessed the differentiation of mISL1-CPCs in histological sections of explanted infarcted hearts 4 weeks after surgery. Since mISL1-CPCs were derived from male ESCs and the host mice were 
A

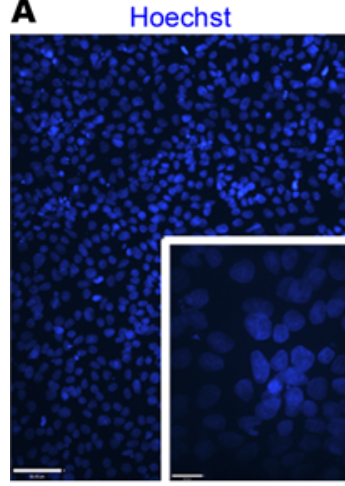

NKX2.5 Hoechst

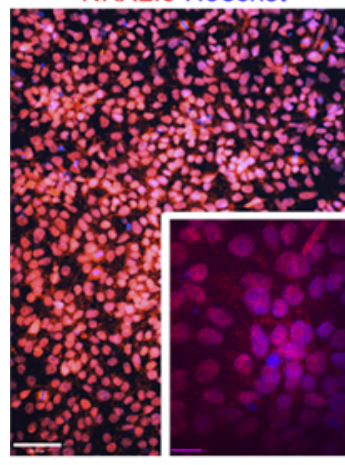

ISL1 Hoechst

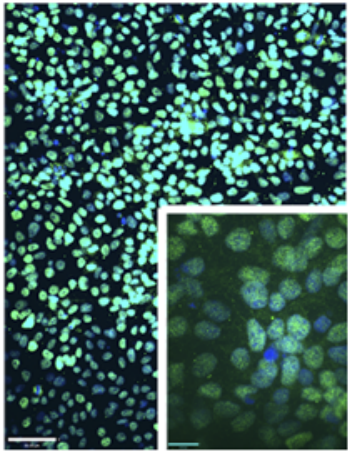

NKX2.5 Hoechst

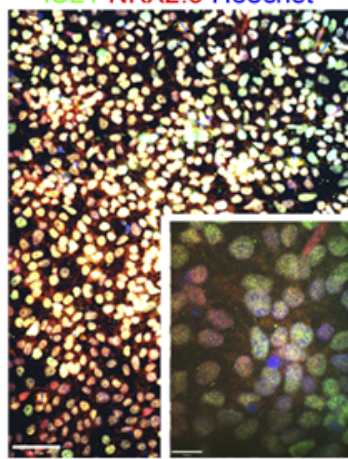

B

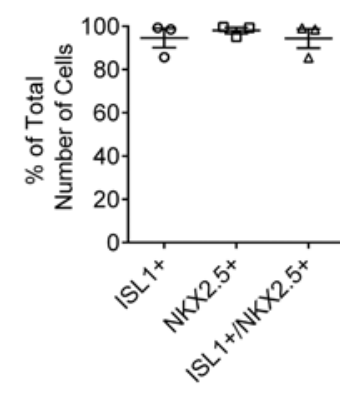

C

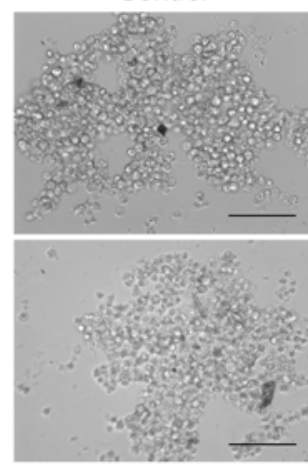

$+Y 27632$

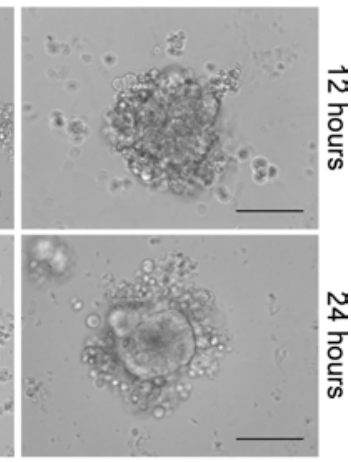

Figure 4. Human ISL1-CPC spheroid formation. (A and B) Day 6 human ISL1-CPC staining for ISL1 (green) and NKX2.5 (red) and quantification (B). Quantifications presented as mean \pm SEM are: ISL1+ $(94.53 \pm 4.42)$; NKX2.5 $(98.04 \pm 1.44)$; ISL1+/NKX2.5+ (94.35 \pm 4.46$)$. Scale bars: $50 \mu \mathrm{m}$ in $\times 20$ pictures and $20 \mu \mathrm{m}$ in $\times 90$ insets. Four random fields per experiment, containing a total of 9,000 cells were quantified. $n=3$. (C) Test for human ISL1-CPC methylcellulose-induced spheroid formation in 96-U well plates with (right panel; +Y27632) and without $5 \mu \mathrm{M}$ ROCK inhibitor Y27632 (left panel; control). Human ISL1-CPC spheroid formation was detected in 96-U well plates with methylcellulose in the presence of Y-27632, 12 hours after plating. Scale bars: $100 \mu \mathrm{m}$. See also Supplemental Figure 5D.

female, we used Y-chromosome fluorescence in situ hybridization (Y-FISH) to detect mISL1-CPC-derived cells. We observed differentiation of mISL1-CPCs into cardiomyocytes marked by Y-FISH and cTnT staining in the scar area (Figure 2, C and E). In contrast, there was no appreciable staining for Y-FISH in the heart of mice injected with Matrigel control (Supplemental Figure 3D), showing the specificity of Y-FISH staining. We also detected ECs derived from mISL1-CPCs in the infarcted hearts, and these CPC-derived ECs appeared to integrate into the host blood vessels (Figure 2, D and E). Consistent with these in vivo data, in vitro differentiation of mISL1-CPCs also revealed the presence of ECs (Supplemental Figure 4) in the absence of additional growth factors. Interestingly, we did not detect SMCs derived from the implanted mISL1-CPCs (Figure 2E), suggesting that the environment in the injured hearts may inhibit SMC differentiation of mISL1-CPCs.

mISL1-CPC spheroids attenuate the reduction of left ventricular (LV) function and decrease the scar size of infarcted mice. To investigate the functional benefits of mISL1-CPC transplantation on contractile function in injured hearts, mice underwent echocardiography 48 hours, 2 weeks, and 4 weeks after surgery without further treatment or with either Matrigel (vehicle control) or mISL1-CPC spheroid treatment. Kaplan-Meier survival analysis revealed that, while treatment of mISL1-CPC did not appear to improve animal survival in comparison with Matrigel treatment $(P=0.28)$, the effect of mISL1-CPC treatment on improving animal survival approached significance compared with no treatment $(P=0.07)$ (Supplemental Figure 5C). The surviving animals were followed up for echocardiographic and histological analyses. All myocardial infarcted groups showed reduced LV fractional shortening at the level of the papillary muscles (M-mode) and LV global ejection fraction on parasternal long-axis view (B-mode) 48 hours after surgery relative to uninjured controls, but there were no differences among the groups with heart injury (Figure 3A). The 2 control groups (no treatment and Matrigel treatment) exhibited a respective $39 \%(P=0.08)$ and $72 \%(P$ $<0.001)$ further reduction of LV fractional shortening, as well as a respective $39 \%(P<0.001)$ and $56 \%$ 
A

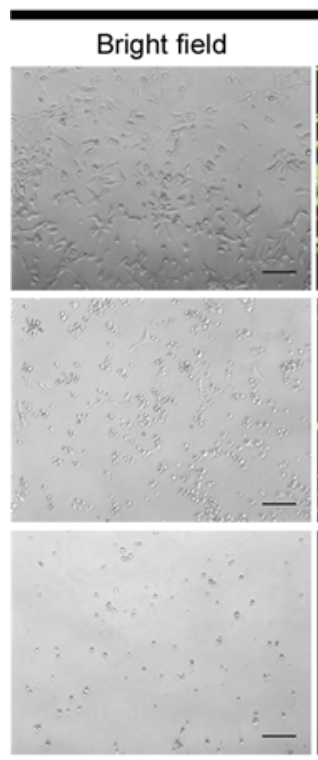

B

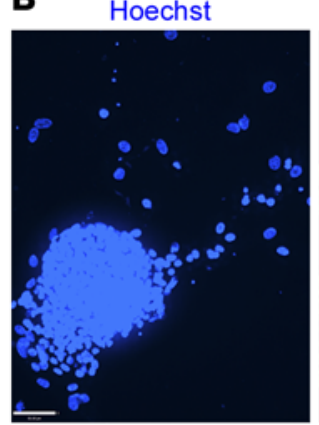

hISL1-CPC single cells
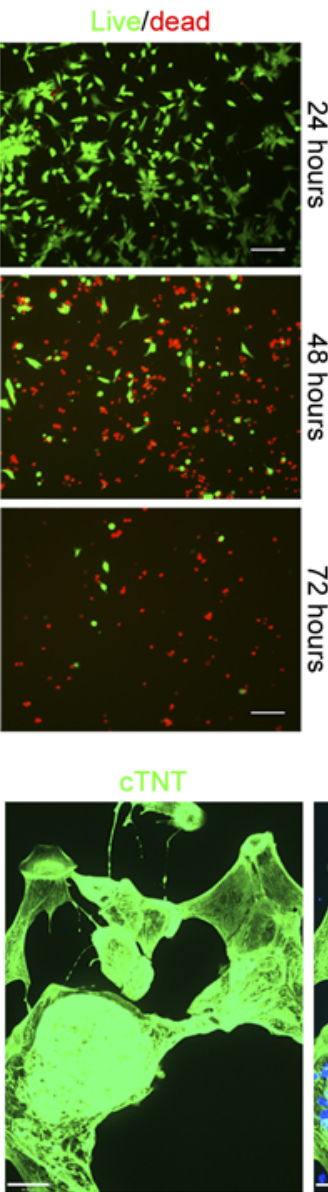

hISL1-CPC spheroids
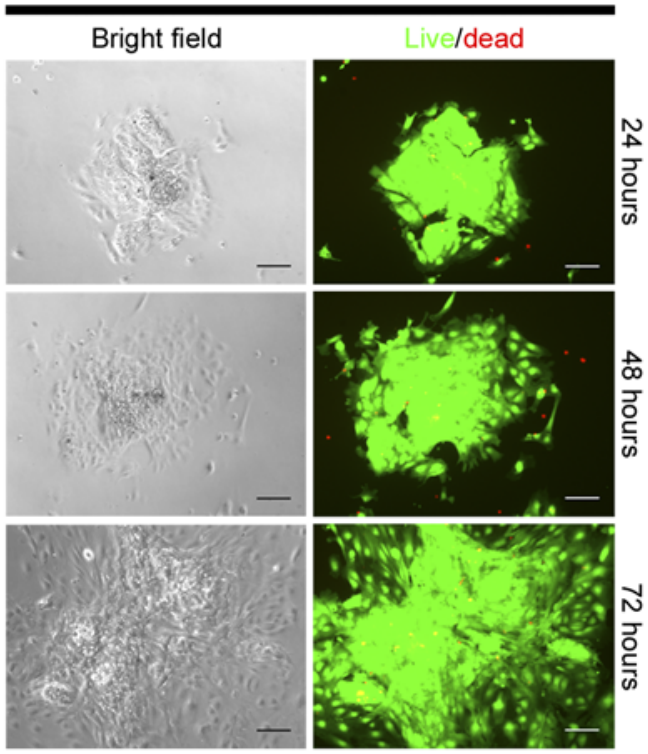
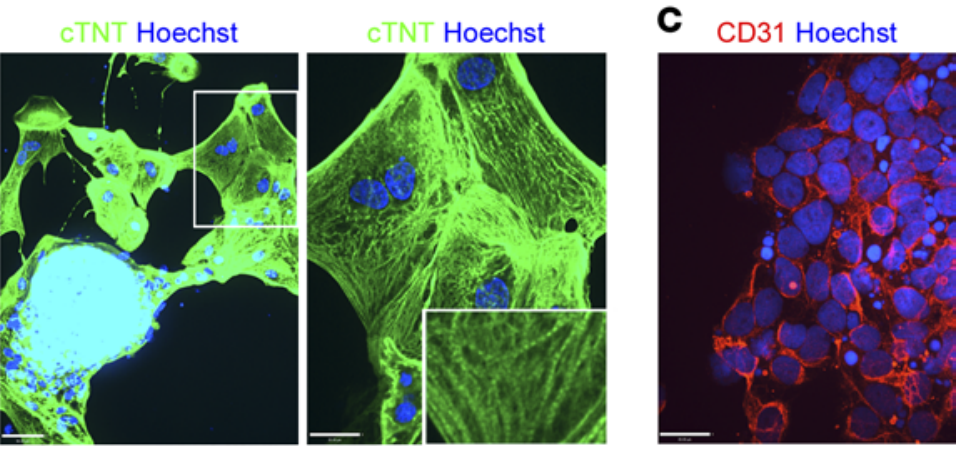

Figure 5. Human ISL1-CPC adhesion and cardiac differentiation. (A) Human ISL1-CPC single cells or ISL1-CPC spheroids were plated on Matrigel-coated plates at a density of $1 \times 10^{5}$ cells/well (24-well plate). Human ISL1-CPC single cells but not spheroids required $5 \mu \mathrm{M}$ Y-27632, which was removed 24 hours after cell plating. While hISL1-CPC spheroids exhibited robust cell survival (green), hISL1-CPC single cell culture showed significant and progressive cell death 48 and 72 hours after seeding (red). Green: calcein staining for live cells. Red: ethidium homodimer staining for dead cells. Scale bars: $100 \mu \mathrm{m}$. (B and C) Human ISL1-CPC spheroids were seeded on Matrigel-coated wells and stained for CTnT (green, B) and CD31 (red, C) 10 days after plating. Scale bars: 50 $\mu \mathrm{m}$ for first, second, and third panels from left in B and $20 \mu \mathrm{m}$ for fourth panel from left in B and panel $\mathbf{C}$. The white rectangle in the third panel from left indicates the area shown in the magnified image displayed in the fourth panel. A magnified inset picture for cTnT is presented to show the sarcomeres in the fourth panel from left in B. See also Movie 3.

$(P=0.01)$ further reduction of LV global ejection fraction, between 48 hours and 4 weeks. However, these functional reductions were markedly attenuated in mISL1-CPC recipient mice: a 25\% (not statistically significant, $P=0.14)$ reduction for fractional shortening and a $23 \%(P<0.05)$ reduction for ejection fraction. Furthermore, mice treated with mISL1-CPCs showed a significantly greater LV fractional shortening (Figure 3A) and global LV ejection fraction (Figure 3, A and B) after 4 weeks than mice receiving Matrigel $(P<$ $0.01)$ or no treatment $(P<0.05)$. Additionally, B-mode analyses also revealed that the mISL1-CPC group showed lower end-diastolic volumes 2 and 4 weeks after treatment compared with the Matrigel group (Figure 3A). In addition, excised hearts of mISL1-CPC-treated mice had reduced LV scar size by Masson's Trichrome staining (Supplemental Figure 6, A and B) and an increased number of CD31+ blood vessels in the scar area when compared with mice treated with Matrigel (Supplemental Figure 6, C, D, and F).

Application of the methylcellulose spherical approach to develop hESC-derived ISL1-CPC spheroids. We next assessed the applicability of our experimental spheroid approach to human cardiac progenitors, utilizing a hESC cardiomyocyte differentiation protocol (16), with 2 modifications. First, we utilized glass chamber slides versus plastic wells used in the original approach; second, we harvested differentiated cells at day 6 that were highly enriched for ISL $1^{+} / \mathrm{NKX} 2.5^{+} \mathrm{CPC}$ versus beating cardiomyocytes harvested at day 14 or later. With this modified approach, we were able to derive highly enriched hISL1-CPCs (hISL1-CPCs, 
A
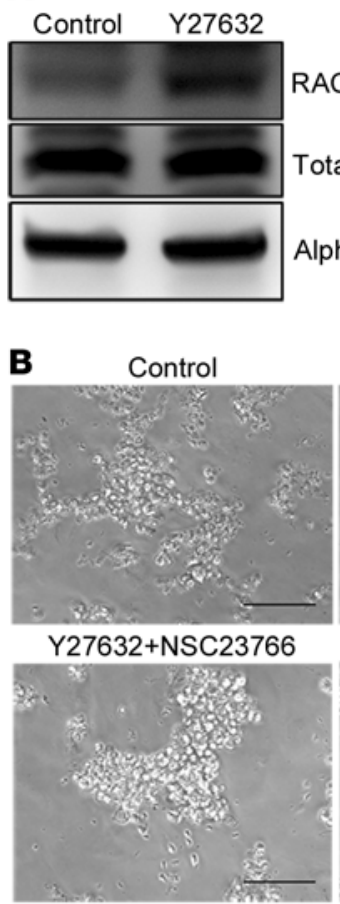

Y27632+U0126

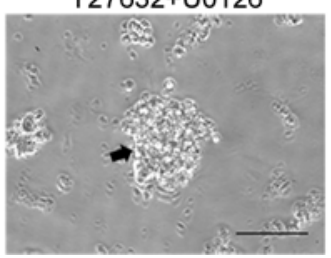

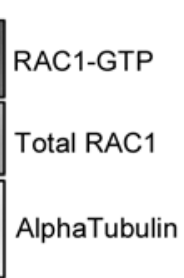

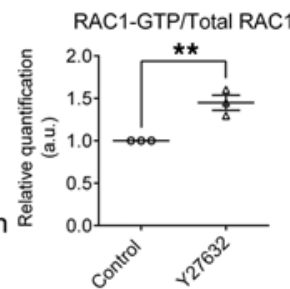

Y27632

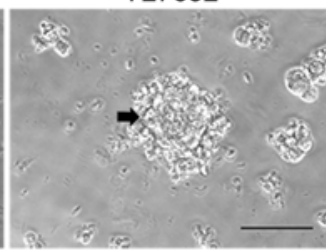

Y27632+BIRB 796

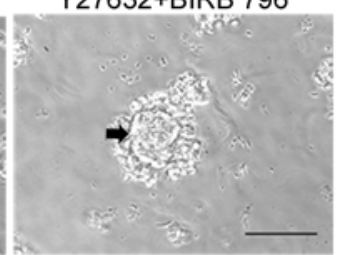

Y27632+SP600125

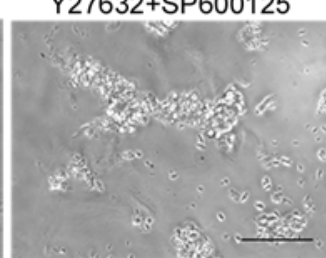

C
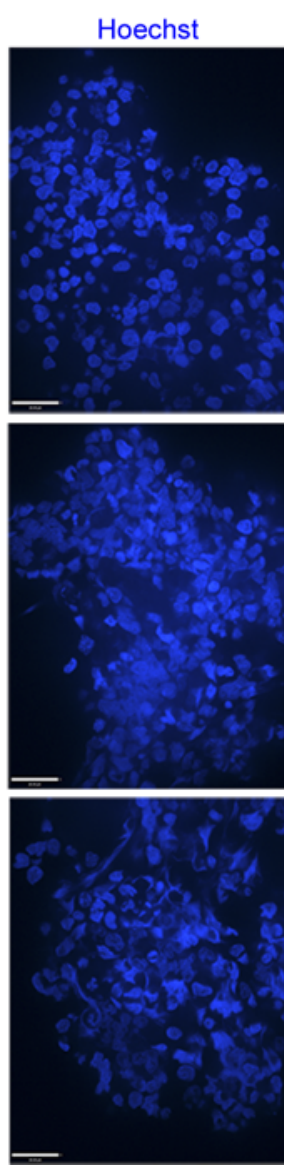
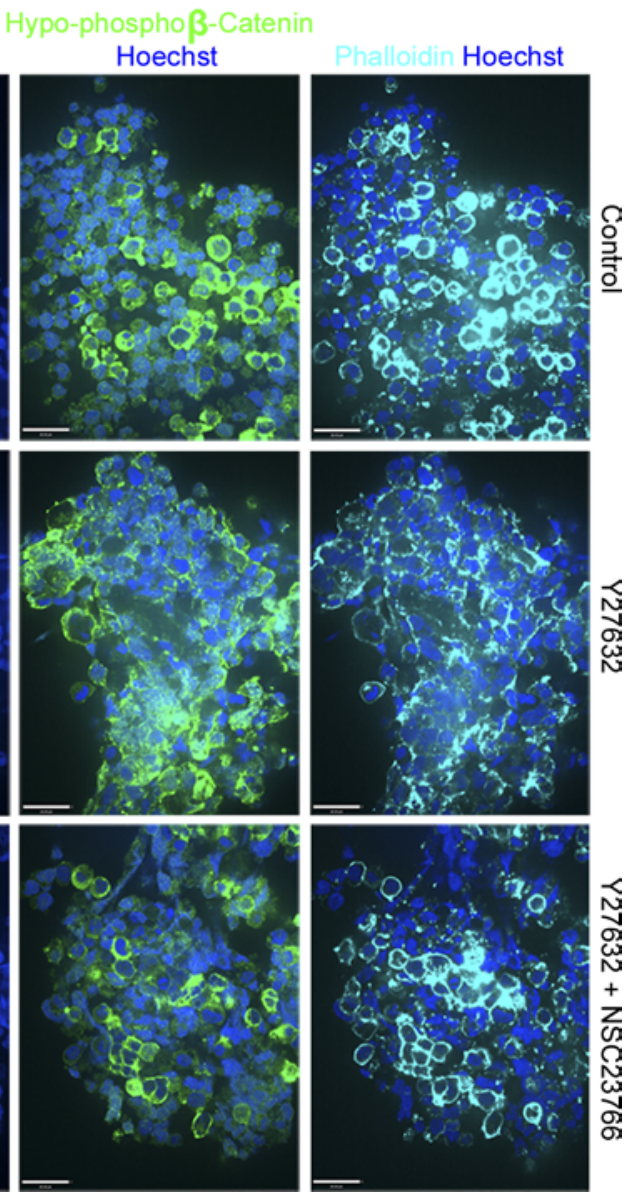

Figure 6. ROCK inhibition by Y-27632 induces RAC1 activation during human ISL1-CPC spheroid formation. (A) RAC1 pull-down with PBD-PAK beads showing active RAC1 (RAC-CTP) in hISL1-CPCs treated with Y27632 during spheroid formation. Cell lysate before pull-down was used to detect total RAC1 and $\alpha$ tubulin (loading control). $n=3$ (unpaired 2-tailed Student's $t$ test, ${ }^{* *} P<0.01$ ). (B) Human ISL1-CPC spheroid formation is abolished in the presence of RAC1 inhibitor (50 $\mu \mathrm{M}$ NSC23766) or JNK inhibitor (10 $\mu \mathrm{M}$ SP600125) but not P38 inhibitor (10 $\mu \mathrm{M}$ BIRB796) or ERK1/2 inhibitor (10 $\mu$ M U0126). Arrows point to formed spheroids. Scale bars: $100 \mu \mathrm{m}$. (C) Staining for hypophospho $\beta$-catenin (green) and phalloidin (cyan) in hISL1-CPCs treated with vehicle control (top panels), Y27632 (middle panels), or Y27632 + NSC23766 (bottom panels) 6 hours after cell plating. Note the rounded morphology in cells treated with control or Y27632 + NSC23766 vs. the spread morphology in cells treated with Y27632 only. Scale bars: $20 \mu \mathrm{m}$.

$94.3 \% \pm 4.5 \%$ ) doubly positive for ISL1 ${ }^{+} / \mathrm{NKX} 2.5^{+}$from hESCs (Figure $4, \mathrm{~A}$ and B). This approach also resulted in the effective derivation of hISL1-CPCs in 2 other pluripotent stem cell lines: the hESC line H7 and an induced pluripotent stem cell (iPSC) line derived from a healthy donor (Supplemental Figure 5D), suggesting the broad applicability of such an approach. The hISL1-CPCs were able to form spheroids in methylcellulose, in the presence of ROCK inhibitor Y27632 (Figure 4C), as early as 12 hours after plating. hISL1-CPC spheroids readily adhered to the Matrigel-coated plates and survived well (Figure 5A). Moreover, these spheroids effectively differentiated into beating cardiomyocytes (Figure 5B and Movie 3 ) and endothelial cells (Figure 5C). In contrast, hISL1-CPC single cells adhered to the Matrigel-coated plates in the presence of Y27632 but started to show marked cell death 48 hours after plating (Figure 5A). These results indicate that hISL1-CPC spheroids have intrinsic cardiac potential and more effective cell survival.

The requirement for Y27632 in hISL1-CPC but not in mISL1-CPC spheroid formation prompted us to investigate its mechanism of action. It has been previously suggested that the RHO GTPases RHO-A and RAC1 may antagonize each other in modulating the actin cytoskeleton (23). Additionally, activation of RAC1 is able to enhance cadherin-mediated cell-cell adhesion (24). We hypothesized that the inhibition of ROCK, a RHO-A effector, by Y27632 would trigger activation of RAC1, leading to augmented cadherin-mediated cell-cell adhesion during spheroid formation. Consistent with this hypothesis, using a RAC1 pull-down assay with $\mathrm{p} 21$ binding domain of $\mathrm{p} 21$ activated kinase 1 (PBD-PAK), which binds to active 
A

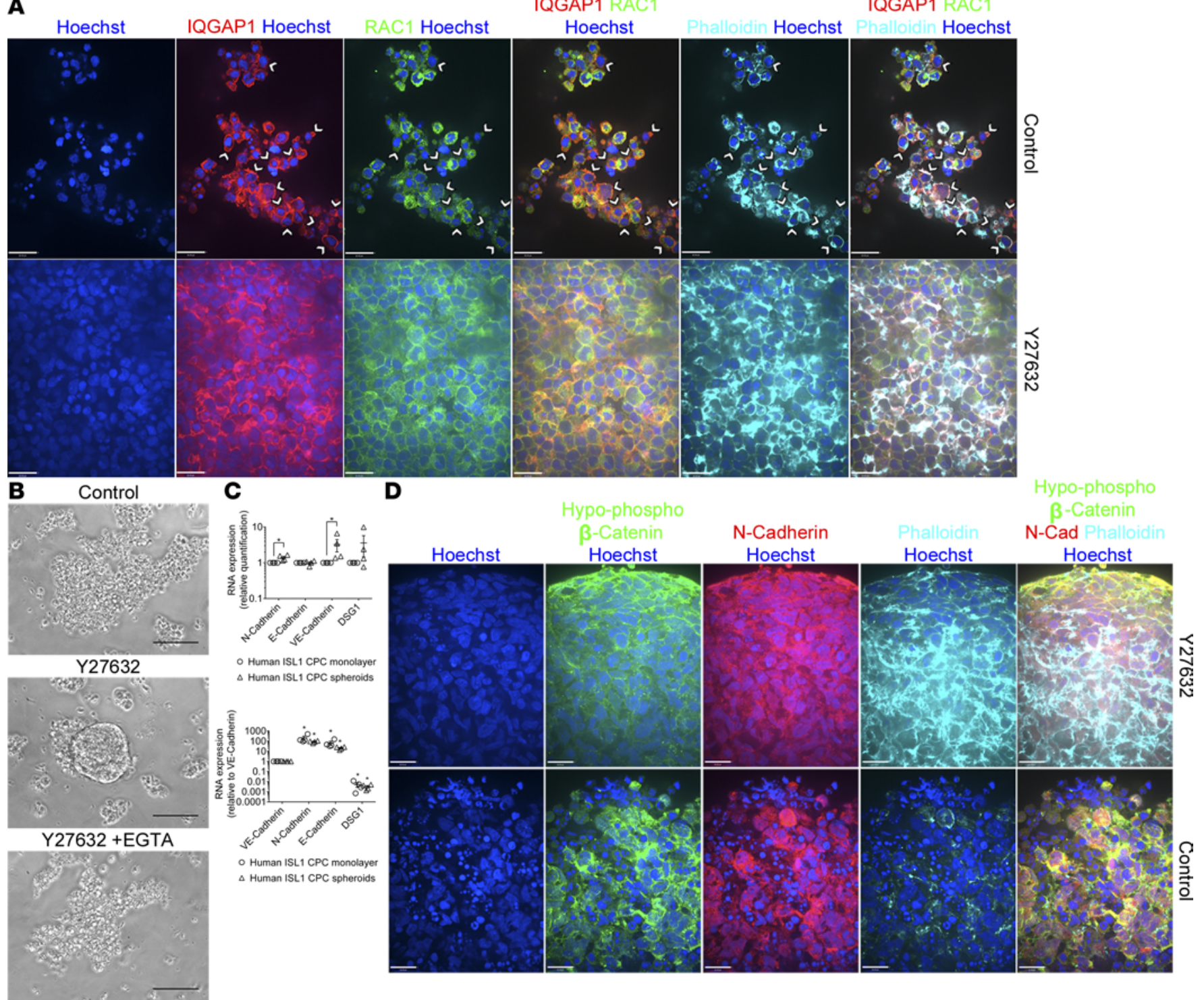

Figure 7. Colocalization of RAC1 and IQGAP1 and cadherin expression during human ISL1-CPC spheroid formation. (A) Staining for RAC1, IQCAP1, and phalloidin in control- or Y27632-treated hISL1-CPCs 2 hours after cell plating during spheroid formation. Note the colocalization of RAC1, IQCAP1, and actin in Y27632-treated cells in the cell-cell contact regions. Arrowheads show the absence of RAC1 in the membrane of cells treated with vehicle control. Scale bars: $20 \mu \mathrm{m}$. (B) Spheroid formation 48 hours after hISL1-CPCs plating in the presence of Y27632 or Y27632 + $2.5 \mathrm{mM}$ EGTA. Scale bar: $100 \mu \mathrm{m}$. (C) Cadherin gene expression by qPCR. DSG1; Desmoglein 1. $n=4$ (Mann-Whitney $U$ test). ${ }^{*} P<0.05$. (D) Staining for $N$-cadherin, hypophospho $\beta$-catenin, and phalloidin in control- or Y27632-treated hISL1-CPCs 8 hours after cell plating during spheroid formation. Scale bars: $20 \mu \mathrm{m}$.

RAC1 (RAC1-GTP), we observed a higher level of RAC1-GTP in Y27632-treated hISL1-CPCs than that in the control sample (Figure 6A). Moreover, in the presence of Y27632, the RAC1 inhibitor NSC23766 prevented hISL1-CPC spheroid formation (Figure 6B). Since JNK is implicated as a downstream mediator of RAC1 (25), the requirement for JNK activation during spheroid formation was investigated. Inhibition of JNK (SP600125) but not P38 (BIRB796) or extracellular signal regulated kinase 1/2 (ERK1/2; U0126) disrupted spheroid formation (Figure 6B).

Additionally, ROCK inhibition by Y27632 led to enhanced cell spreading, accompanied by a reorganization of the actin cytoskeleton shown by phalloidin staining, compared with control or double-inhibition of ROCK and RAC1 (Figure 6C). Activated RAC1 has previously been shown to facilitate cadherin-mediated cell-cell adhesion by binding to IQGAP1 and crosslinking actin filaments at the cell-cell contact regions (24). We thus examined subcellular localization of RAC1 and IQGAP1 and observed a strong colocalization of RAC1, IQGAP1, and actin filaments at the cell-cell contact regions in hISL1-CPCs treated 


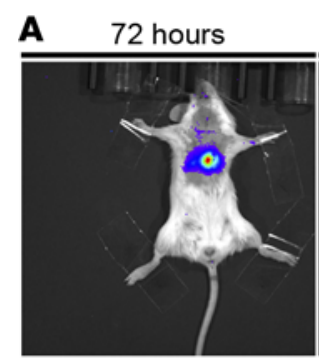

C TNT HLA Hoechst
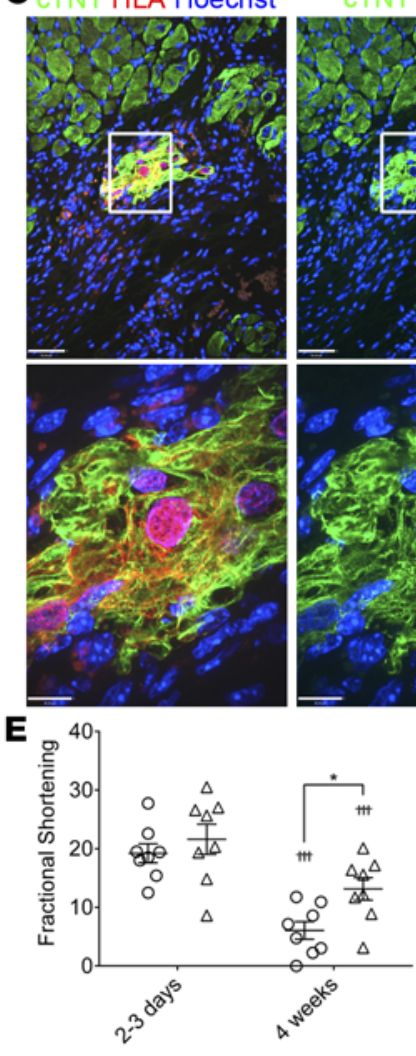

2 weeks
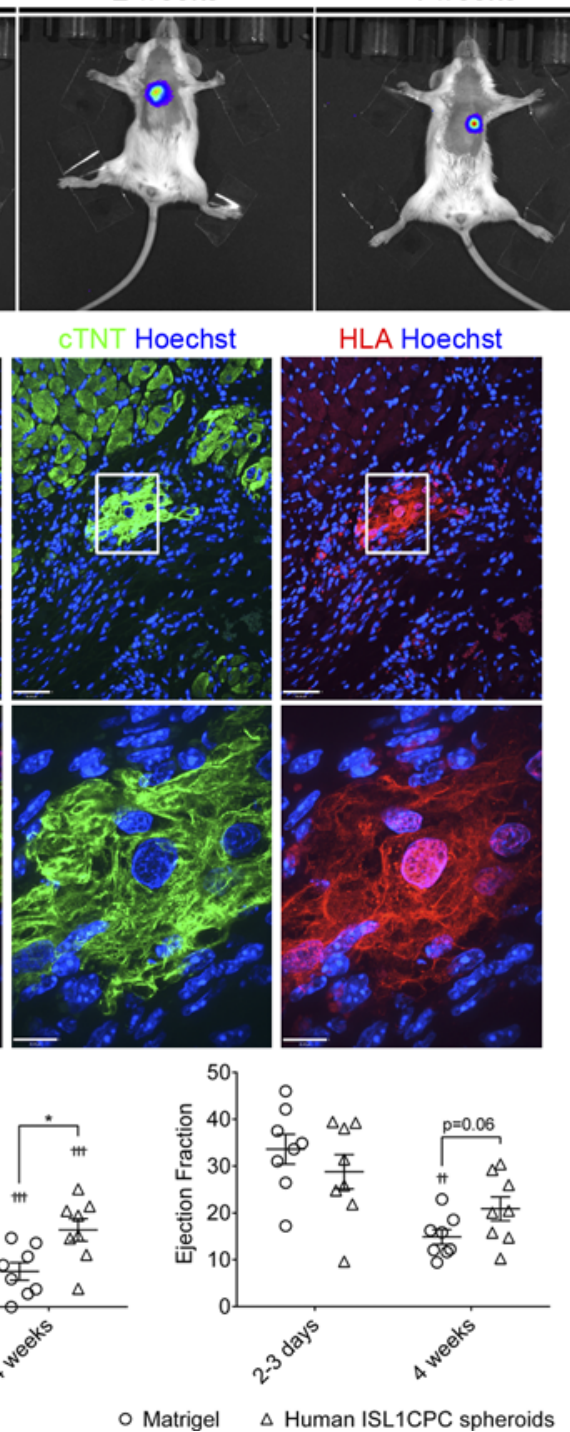

Luciferase In vivo bioluminescence quantification

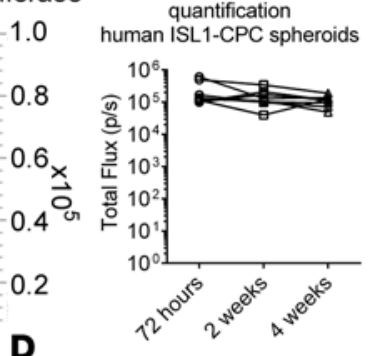

B

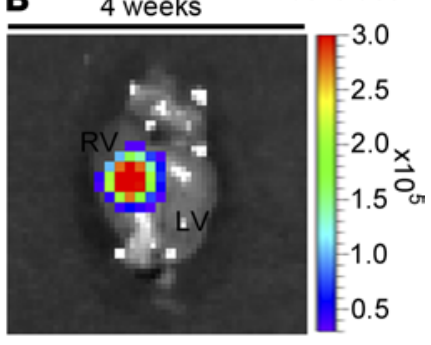

\section{HLA Hoechst D}

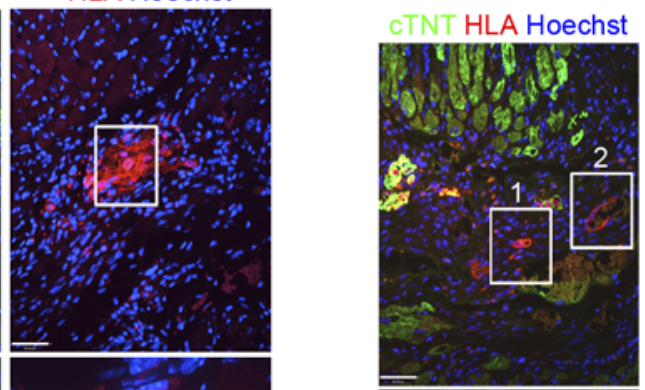

CTNT Hoechst

HLA Hoechst
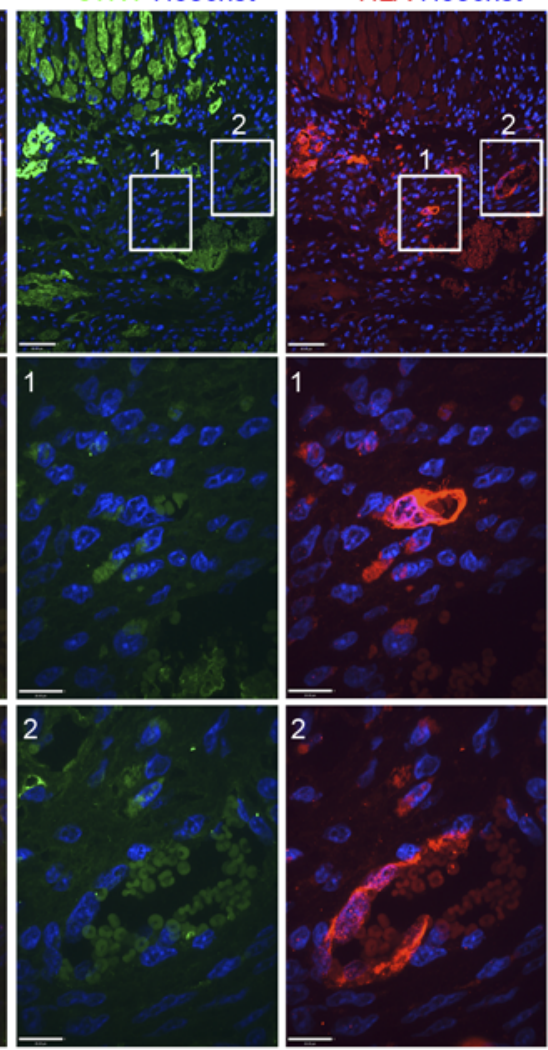

Figure 8. Human ISL1-CPC spheroid-derived cells in infarcted hearts. (A) Bioluminescence (BLI) imaging recorded in 1 mouse at 3 different time points after left anterior coronary artery ligation and human ISL1-CPC spheroid injection. Graph shows BLI quantification at 72 hours, 2 weeks, and 4 weeks after cell delivery. $n=8$. Statistical analysis performed with 1-way repeated measurements ANOVA with Greenhouse-Geisser correction $(P=0.14)$. (B) Explanted heart 4 weeks after surgery and human ISL1-CPC spheroid injection showing BLI in the peri-infarct area. LV, left ventricle; RV, right ventricle. In $\mathbf{A}$ and $\mathbf{B}$, the numbers in the scale bars were introduced manually to enlarge the font size. (C and D) Histological analysis of a heart, 4 weeks after MI. (C) Human ISL1-CPC spheroids differentiated into CMs in the midst of the scar. The white rectangle indicates the area shown in the magnified image displayed below. Scale bars: $50 \mu \mathrm{m}$ (top panels) and $20 \mu \mathrm{m}$ (bottom panels). (D) Human ISL1-CPC spheroids differentiated into functional blood vessels. The images marked by the numbers in the middle and bottom panels represent the magnified area demarcated by the rectangles in the top panels. HLA, human leukocyte antigen, which marks human ISL1-CPC-derived cells. Scale bars: $50 \mu \mathrm{m}$ (top panels) and $20 \mu \mathrm{m}$ (middle and bottom panels). (E) M-Mode fractional shortening recorded along the mid-left ventricular short axis, and B-Mode ejection fraction recorded along the parasternal long axis of mice subjected to ligation of the left coronary artery. Matrigel injection and human ISL1-CPC spheroid injection ( $n=8$ mice). Note that Matrigel-injected animals included six mice used in mouse ISL1-CPC studies. Three measurements were averaged per animal for each time point to obtain the M-Mode data. Samples were analyzed by 2-way ANOVA and pair comparisons using unpaired 2-tailed Student's $t$ test between groups ( ${ }^{*} P<0.05$ ), as well as pair comparisons for time points ( $2-3$ days vs. 4 weeks) within the same group using paired 2-tailed Student's $t$ test $\left({ }^{\dagger+} P<0.01\right.$; $\left.{ }^{\dagger+} P<0.001\right)$. See also Supplemental Figure 5 , B and C, and Figures 6 and 7.

with Y27632 (Figure 7A). This result suggests that RAC1-IQGAP1 interaction may play an important role during Y27632-activated spheroid formation. In addition, EGTA treatment disrupted the formation of spheroids (Figure 7B), suggesting that calcium-dependent cadherin activity may be necessary for spheroid formation. We next investigated whether ROCK inhibition may result in induction of cadherin expression during hISL1-CPC spheroid formation. N-cadherin appeared to be the most abundant cadherin and was significantly upregulated during ROCK inhibition-induced spheroid formation compared 
with that in monolayer culture (Figure 7C). Furthermore, efficient adherens junctions marked by the presence of $\mathrm{N}$-cadherin, $\beta$-catenin, and actin filaments were present in hISL1-CPCs treated with Y27632, in striking contrast to cells treated with vehicle control (Figure 7D). In summary, these results suggest that RAC1 activation triggered by ROCK inhibition may enhance cadherin-mediated cell-cell adhesion during hISL1-CPC spheroid formation.

hISL1-CPC spheroids engraft, survive, differentiate to cardiomyocytes, and form functional blood vessels within infarcted hearts. To analyze the reparative capacity of hISL1-CPCs after MI, we generated spheroids in 12-14 hours in the presence of methylcellulose and Y27632. Two thousand spheroids (2 million hISL1-CPCs) were injected per mouse $(n=8)$ in the peri-infarct area of the heart in SCID-bg females after permanent ligation of the left coronary artery. hISL1-CPC spheroid-derived cells were readily detected by bioluminescence at 3 different time points analyzed - 72 hours, 2 weeks, and 4 weeks in vivo (Figure 8A and Supplemental Figure 3C) - as well as in the explanted hearts ex vivo (Figure 8B). There was no significant decrease in the magnitude of the bioluminescence signals during the course of 4 weeks (one-way repeated measurements ANOVA, $P=0.14$ ), suggesting that hISL1-CPCs injected as spheroids survived effectively following injection into the infarcted heart. Histological analyses 4 weeks after injection using class I HLA or human nuclear antigen $(\mathrm{HuNu})$ staining, revealed that hISL1-CPC spheroids differentiated primarily into cardiomyocytes in the infarcted hearts (Figure 8C and Supplemental Figure 6G). Additionally, hISL1-CPC spheroid-derived cells formed functional (note the presence of rbcs inside) small vessels solely composed of hECs (Figure 8D and Supplemental Figure 7) as well as functional chimeric vessels containing both human and mouse ECs (Figure 8D). Notably, we did not detect teratomas in any of the hearts analyzed.

Alleviation of LV functional decrease, reduced infarct size, and increased blood vessel formation in hISL1-CPC spheroid-injected hearts. LV function was recorded by echocardiography at 2-3 days and 4 weeks after MI and cell delivery. We first examined LV function in infarcted mice receiving either Matrigel vehicle control or hISL1-CPC spheroid treatment by measuring LV fractional shortening at the level of the papillary muscles (M-mode) and global ejection fraction on parasternal long-axis view (B-mode) 2-3 days after surgery. There did not appear to be appreciable differences between the $2 \mathrm{MI}$ groups (Figure $8 \mathrm{E}$ ). Notably, although Matrigel-treated animals exhibited a respective $70 \%$ and 50\% further reduction of LV fractional shortening $(P<$ $0.001)$ and global LV ejection fraction $(P<0.01)$ between day $2-3$ and 4 weeks, these reductions were largely attenuated in hISL1-CPC-treated animals (a 39\% reduction for fractional shortening, $P<0.001$, and a $28 \%$ reduction for ejection fraction, not statistically significant, $P=0.09$ ). Furthermore, mice treated with hISL1CPC spheroids showed an increased LV fractional shortening $(P=0.01)$ and global ejection fraction $(P=$ 0.06) (Figure $8 \mathrm{E}$ ) after 4 weeks, compared with those injected with Matrigel control. These results suggest that hISL1-CPCs are capable of attenuating the deterioration of global LV contractile function in the infarcted hearts. In addition, excised hearts of hISL1-CPC spheroid-treated mice showed a 2.6- and 1.7-fold (length measurements) and a 2.6- and 1.4-fold (area measurements) reduced scar size by Masson's Trichrome staining, in comparison with mice injected with Matrigel and mISL1-CPCs, respectively (Supplemental Figure 6 , $\mathrm{A}$ and B). hISL1-CPC treatment also led to a 2.6- and 1.6-fold increased number of CD31 ${ }^{+}$blood vessels in the scar area compared with Matrigel and mISL1-CPC treatment, respectively (Supplemental Figure 6, C-F). Additionally, mice receiving hISL1-CPCs showed a significantly higher survival rate than those receiving no treatment $(P<0.05)$ (Supplemental Figure 5C). There also appeared to be a trend of increased survival for animals receiving hISL1-CPC treatment compared with those that received Matrigel control $(P=0.14)$.

Host cardiomyocytes contain active YAP in hISL1-CPC spheroid-injected hearts. Since hearts injected with hISL1-CPC spheroids showed a considerable decrease in scar size, we hypothesized that hISL1-CPC spheroids might exert a proliferative and/or protective effect on host myocardium. Recently, the Hippo pathway effector Yes-associated protein (YAP) has been implicated in activating the PI3K-AKT signaling pathway, leading to an induction of proliferation and survival of cardiomyocytes after MI (26). Histological analysis of the excised hearts revealed a 4.5-fold increase of host cardiomyocytes with activated (nuclear) YAP in the group injected with hISL1-CPC spheroids compared with those in Matrigel-injected group (Figure 9). However, there was no clear correlation between cardiomyocytes with activated YAP and BrdU or KI67 (Supplemental Figure 8A). Notably, we did observe that cardiomyocytes with activated YAP showed a high level of expression of phosphorylated ribosomal subunit S6 (p-S6), the target of prosurvival/anabolic $\mathrm{PI} 3 \mathrm{~K} / \mathrm{AKT} / \mathrm{mTOR}$ pathway (Supplemental Figure 8B). These results raise the possibility that hISL1-CPC spheroids do not induce the proliferation of cardiomyocytes in the border zone of the infarct but may protect them by activating YAP and triggering the prosurvival/anabolic PI3K/AKT/mTOR pathway. 
A
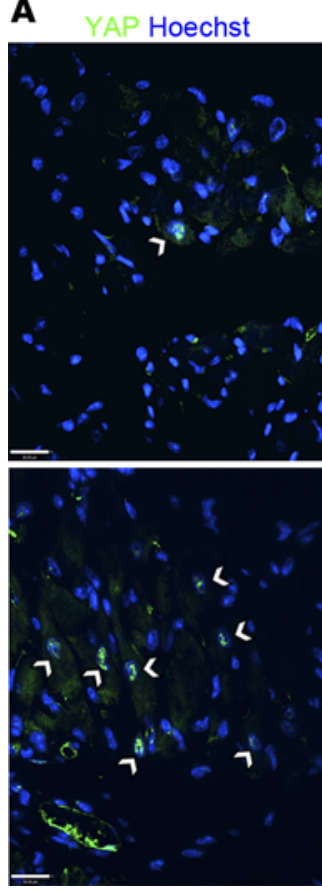

cTNT Hoechst
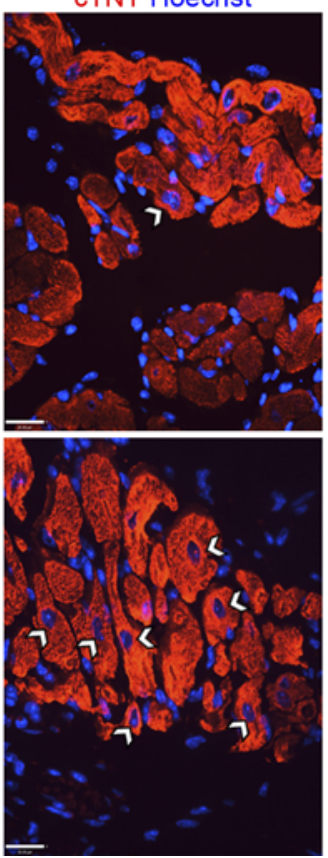

cTNT YAP Hoechst
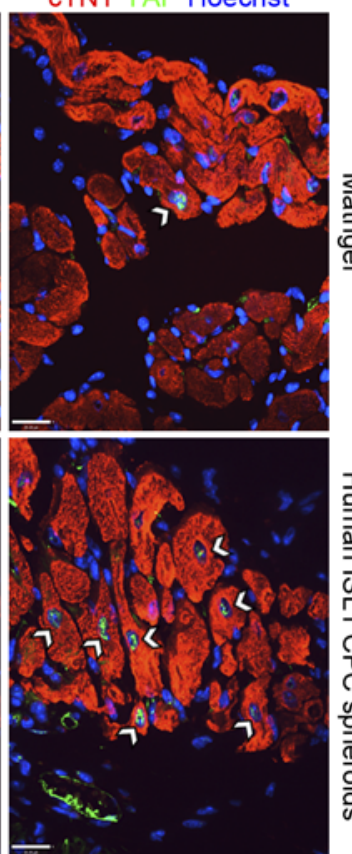

B

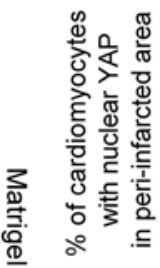

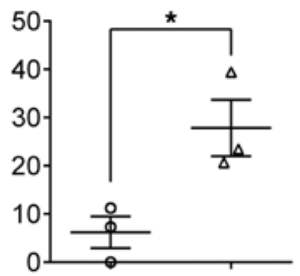

- Matrigel

$\Delta$ Human ISL1 CPC spheroids

Figure 9. Induction of YAP activation in host cardiomyocytes by human ISL1-CPC spheroids. (A) Heart section of a representative mouse injected with Matrigel (top panels) or human ISL1-CPC spheroids (bottom panels) showing nuclear (active) YAP in host cardiomyocytes. Scale bar: $20 \mu \mathrm{m}$. (B) Graph representing host cardiomyocytes with active YAP. Two sections, in the mid-part of the heart and close to ligation, were quantified per heart. $n=3$ hearts per experimental condition (unpaired 2-tailed Student's $t$ test).

${ }^{*} P<0.05$. See also Supplemental Figure $8, A$ and $B$.

Growth factors expressed in hISL1-CPC spheroids induce YAP activation and protect neonatal rat cardiomyocytes against hypoxia and serum and glucose deprivation. To identify the potential growth factor(s) or cytokine(s) involved in cardiac protection mediated by hISL1-CPC spheroids, we screened by quantitative PCR (qPCR) the mRNA expression of multiple candidate genes (Figure 10A and Supplemental Figure 8C). Expression of Neuregulin $1 \beta$ (NRG1 $\beta$ ) and Vascular endothelial growth factor $c$ (VEGF-C) showed a respective 22- and 13-fold increase in hISL1-CPC spheroids compared with that in undifferentiated hESCs (Figure 10A). Interestingly, the spheroid formation also induced the expression of Angiopoietin 1 (ANGPT1) in hISL1-CPC spheroids, showing a 14-fold increase in comparison with that in hISL1-CPCs monolayer or undifferentiated hESC culture (Figure 10A). Additionally, spheroids formation of hESC-derived cardiomyocytes (hESC-CMs) also resulted in significantly higher levels of expression of NRG1 $\beta, V E G F-C$, and ANGPT1 than those in undifferentiated hESCs (Supplemental Figure 8, D and G). Previous studies reveal that NRG1 $\beta$ can induce YAP activation via the epidermal growth factor receptor (EGFR/ERBB) (27). hISL1-CPC spheroid-derived conditioned medium induced the activation of YAP, shown by a significant reduction in the ratio of phospho- to total YAP, in neonatal rat cardiomyocytes under hypoxia and serum deprivation (Figure 10B). YAP activation was reversed when the ERBB inhibitor Lapatinib was included in the medium (Figure 10B), suggesting that NRG1 $\beta$ or other ERBB ligands might be involved in YAP activation. Moreover, exogenous NRG1 $\beta$ induced YAP nuclear translocation and AKT activation in neonatal rat cardiomyocytes under hypoxia and serum and glucose deprivation (Figure 10, C-E). Additionally, NRG1 $\beta$ induced AKT activation in human induced pluripotent stem cell-derived cardiomyocytes under hypoxia and serum and glucose deprivation (Supplemental Figure 8, E and F). These results suggest that NRG1 $\beta$ may protect cardiomyocytes from ischemic conditions by inducing YAP and AKT.

Neuregulin 1 and angiopoietin 1 reduce fibrosis in human cardiac fibroblasts. The reduced scar formation in the hearts of mice injected with hISL1-CPC spheroids with respect to the Matrigel-injected group (Supplemental Figure 6, A and B) could be attributed to paracrine factors inhibiting myofibroblast activation and collagenous matrix deposition. To test this hypothesis, we analyzed the effect of NRG1 $\beta$, VEGF-C, and ANGPT1 in a TGF- $\beta 1$-induced model of fibrosis using adult human cardiac fibroblasts. NRG1 $\beta$ and ANGPT1 significantly decreased TGF- $\beta 1$-induced fibrosis, shown by a decreased number of cells with SMA filament bundle formation (Figure 11A) and collagen 1a1 production (Figure 11B). Additionally, NRG1 $\beta$-treated cells also resulted in a significant reduction of the expression of SMA that was induced by TGF- $\beta 1$ (Figure 11B), suggesting an antagonistic action of NRG1 $\beta$ toward TGF- $\beta 1-$ induced cardiac fibrosis. 
A

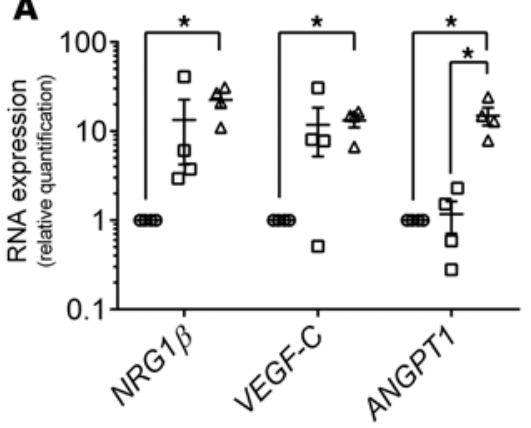

- Human ESCs

- Human ISL1 CPC monolayer

$\triangle$ Human ISL1 CPC spheroids
B

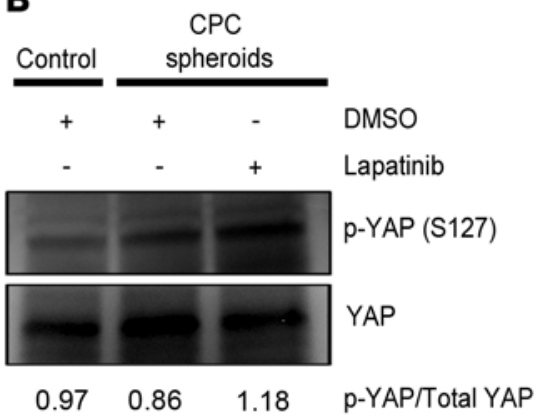

D Nuclear YAP
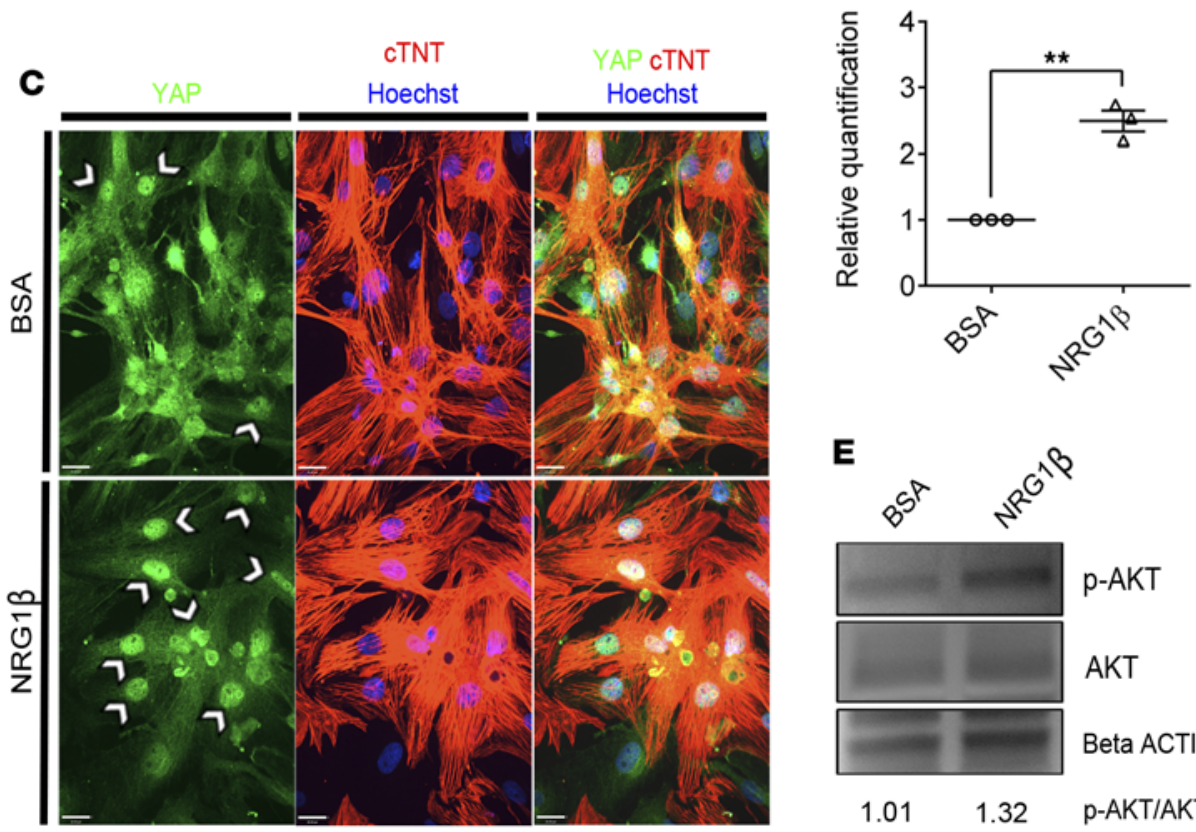

E

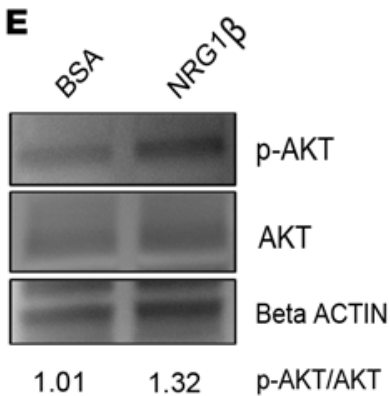

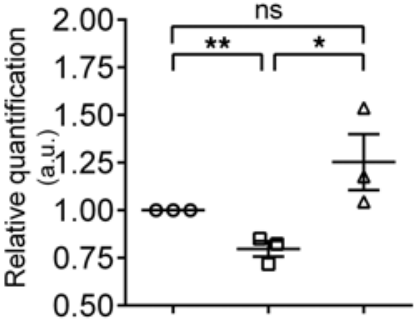

- Control $\mathrm{CM}+\mathrm{DMSO}$

- CPC spheroids CM + DMSO

$\triangle$ CPC spheroids $\mathrm{CM}+$ Lapatinib

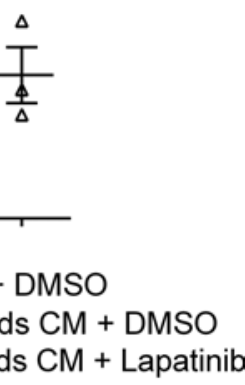

pYAP/Total YAP

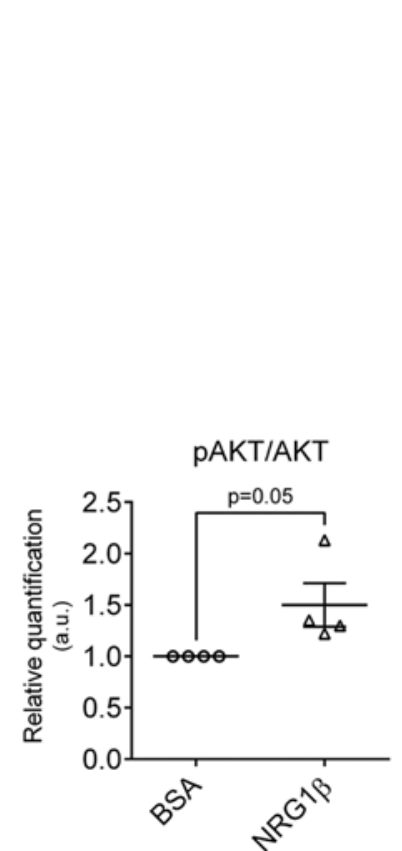

Figure 10. Human ISL1-CPC spheroids produced growth factors with cardio-protective properties. (A) qPCR showing an upregulation of the expression of Neuregulin $1 \beta$ (NRG1ß), Vascular endothelial growth factor C (VEGF-C), and Angiopoietin 1 (ANGPT1) in hISL1-CPC spheroids. $n=4$ (Mann-Whitney $U$ test). ${ }^{*} P<0.05$. (B) Representative Western blots and graph showing increased levels of active YAP (lower $p$-YAP/Total YAP ratio) in neonatal rat cardiomyocytes cultured under hypoxia and serum starvation in the presence of hISL1-CPC conditioned medium (CM). YAP activation was abolished when cardiomyocytes were treated with the ERBB inhibitor Lapatinib. $n=3$ (unpaired 2-tailed Student's $t$ test, ${ }^{*} P<0.05,{ }^{*} P<0.01$ ). (C and D) NRG1 $\beta$ induces YAP activation (nuclear translocation) in neonatal rat cardiomyocytes under hypoxia and serum deprivation. Arrows indicate cardiomyocytes with nuclear YAP. Scale bars: $20 \mu \mathrm{m}$. Graph representing quantification of cardiomyocytes containing nuclear YAP normalized by total number of cardiomyocytes. $n=3$ (unpaired 2-tailed Student's $t$ test, ${ }^{* *} P<0.01$ ). (E) Representative Western Blots and graph quantification showing NRG1-mediated activation of AKT in neonatal rat cardiomyocytes under hypoxia and glucose and serum deprivation. $n=4$ (unpaired 2-tailed Student's $t$ test). See also Supplemental Figure 8, C-F.

\section{Discussion}

The current study is the first reported use of mouse and human ISL1-CPCs for cardiac repair. ISL1-CPCs are a CPC population with authentic cardiac origin based on our previous detailed lineage tracing studies $(9,11)$. Our experiments are also the first to use a methylcellulose-based cell spheroid approach for cardiac repair. mISL1-CPC spheroids markedly enhanced cardiomyocyte differentiation, and mouse and human ISL1-CPC spheroid-derived cells engrafted effectively 4 weeks after injection, having differentiated into both cardiomyocytes and ECs. Importantly, ISL1-CPC spheroid transplantation attenuated the deterioration of LV contractile function, reduced scar size, and increased blood vessel formation. Moreover, our results show that the methylcellulose spheroid approach maintains the intrinsic cardiac potential of hISL1CPCs and promotes superior cell survival compared with a monolayer culture strategy. Thus, the ISL1-CPC 

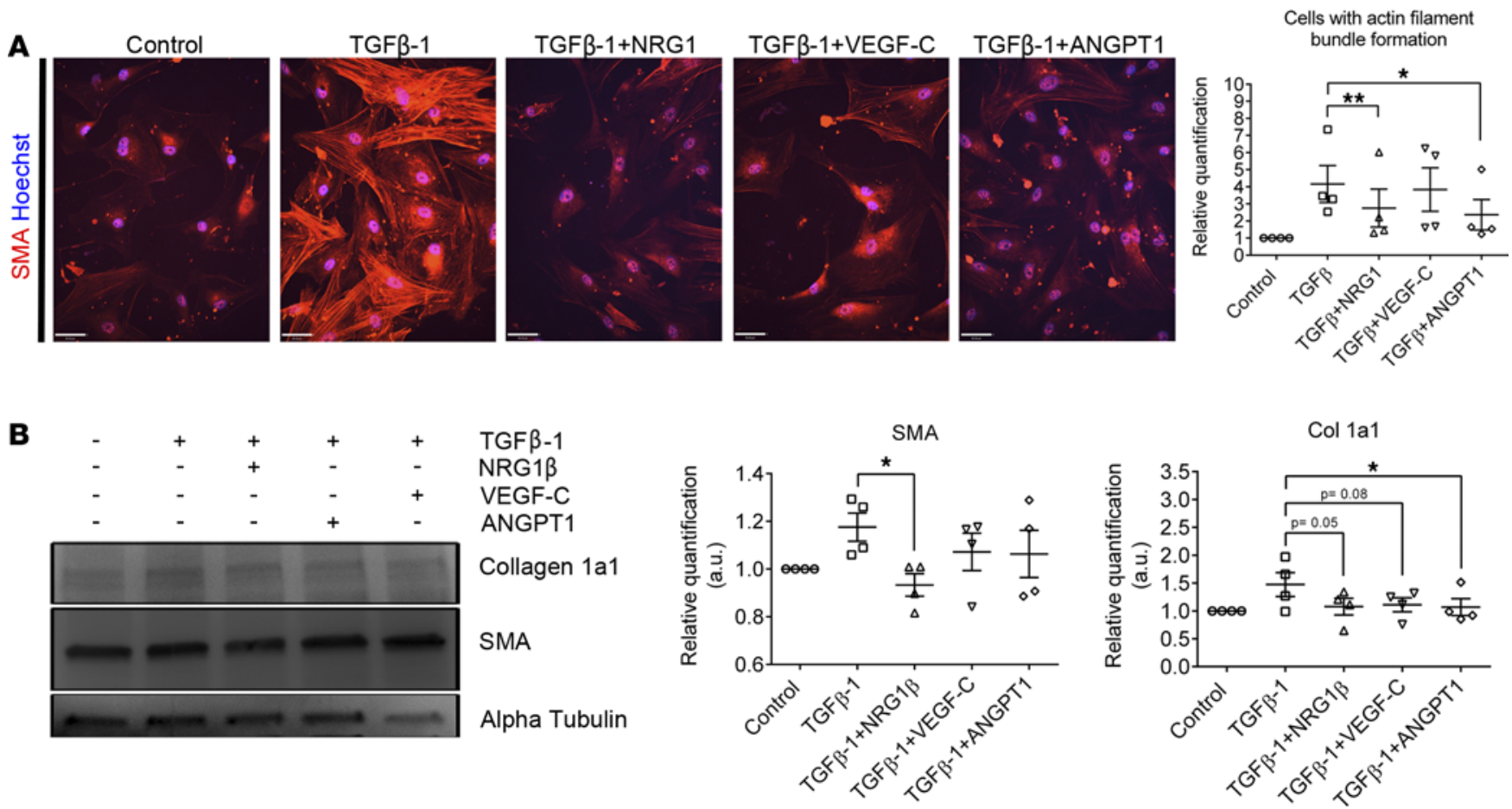

Figure 11. Human ISL1-CPC spheroids produced growth factors with antifibrotic properties. (A) SMA staining in human adult ventricular cardiac fibroblasts, 48 hours after treatment with the indicated growth factors. Scale bars: $50 \mu \mathrm{m}$. Graph showing reduced numbers of human cardiac fibroblasts with SMA ${ }^{+}$filament bundle formation, 48 hours after treatment with NRG1 $\beta$ or ANGPT1. $n=4$ (paired 2-tailed Student's $t$ test, ${ }^{*} P<0.05$, ${ }^{* *} P<0.01$ ). (B) Western blots and graph quantification showing that NRG1 $\beta$ and ANGPT1 reduced collagen 1a1 protein and that NRG1 $\beta$ reduced SMA in human adult ventricular cardiac fibroblasts. $n=4$ (paired 2-tailed Student's $t$ test, ${ }^{*} P<0.05$ ).

spheroid approach combines the use of a valuable cell type with authentic cardiac differentiation potential, with a robust delivery approach that enhances cardiomyocyte differentiation and cell engraftment.

Implantation of cardiospheres formed on poly-D-lysine-coated dishes with cells derived from adult atrial tissue has similarly been found to increase cell engraftment and cardiac function after MI (5). However, these cardiospheres contain cells with low cardiomyocyte differentiation potential and are time-consuming to derive (3-6 weeks) $(5,17)$. In contrast, our ISL1-CPC spheroids formed in methylcellulose were rapidly generated (12-24 hours) and showed robust cardiomyocyte differentiation in vitro and in vivo. Methylcellulose appears to be essential for spheroid formation, since ISL1-CPCs failed to aggregate into spheroids in the absence of this inert viscosity-modulating substance. ISL1-CPC spheroids showed persistent engraftment, as evidenced by the magnitude of the bioluminescence signals that was comparable 3 and 28 days after implantation. In contrast, CPCs delivered in conventional, single cell suspension had an engraftment efficiency of $1.4 \%$ when assessed 28 days (28) after implantation. Importantly, the methylcellulose spheroid approach can be applied to many cell types, such as ISL1-CPCs (this study), ESCs, cardiomyocytes, ECs (Supplemental Figure 8G), ESC-derived neuronal progenitors, and GABAergic neurons (data not shown), thereby potentially providing a general cell delivery platform to enhance cell survival/ engraftment during cell-based regenerative therapies.

Primary heart tissue-derived CPCs have beneficial properties in the injured heart, but their derivation may be limited operationally due to tissue accessibility. In contrast, CPCs derived from ESCs could represent a valuable cell source for cardiac repair based on their unlimited accessibility. Indeed, we can derive an unlimited number of murine and human ISL1-CPCs that readily differentiate into cardiomyocytes by highly effective ESC differentiation approaches. Thus, a critical aspect of our strategy is that it provides a CPC source with true cardiac potential and unlimited accessibility.

Due to the small size of the murine heart that prevents cell injection at multiple locations, ISL1-CPC spheroid-derived cardiomyocytes appeared to reside in the midst of the scar tissue distant from host cardiomyocytes. Together with our observations that ISL1-CPC spheroids differentiated well into cardiomyocytes in vitro, our results suggest that ISL1-CPC-derived cardiomyocytes were most likely not the result of cell 
fusion with host endogenous cardiomyocytes. Future studies will be required in more clinically relevant larger animal models that are more amenable to the delivery of cells at multiple locations, which might further enhance engraftment and the functional coupling between host and donor cells.

In this study, there did not appear to be sufficient functional coupling between donor and host cardiomyocytes by 28 days after treatment. However, the ISL1-CPC-derived cardiac cells were viable with a persistent engraftment between days 3 and 28, and a longer study might help to reveal potential functional coupling between donor and host cardiomyocytes. It is likely that ISL1-CPC-derived cardiomyocytes may also help to reduce pathological remodeling in the injured hearts via a paracrine effect. Additionally, increased blood vessel formation in the ISL1-CPC spheroid-treated mice will likely contribute to the early beneficial effects on heart function.

We observed that hISL1-CPC spheroid injection resulted in a significantly increased number of host cardiomyocytes with activated YAP and a high level of expression of p-S6, raising the possibility that hISL1-CPC-derived growth factor(s) may protect host cardiomyocytes from ischemia by activating YAP and triggering the prosurvival/anabolic PI3K/AKT pathway. Consistent with this notion, NRG1 $\beta$ was highly upregulated in hISL1-CPC spheroid culture, and hISL1-CPC spheroid conditioned medium or recombinant NRG1 $\beta$ appeared to activate YAP and/or AKT in neonatal rat cardiomyocytes under hypoxia. In agreement with NRG1 $\beta$ in reducing fibrotic response in rodent cardiac fibroblasts (29), we found that NRG1 $\beta$ was able to reduce TGF- $\beta$-induced fibrosis in human adult ventricular cardiac fibroblasts. It is likely that NRG1 $\beta$ derived from injected hISL1-CPC spheroids might also enhance cardiac function by inhibiting pathologic cardiac fibrosis. Future endeavors will be made to further dissect how NRG1 $\beta$ regulate cardiomyocyte survival and cardiac fibrosis during ischemic insult.

Recent studies showed that human ESC- or iPSC-derived cardiomyocytes have promise for effective cardiac repair (30-32). The potential of ESC- or iPSC-derived CPCs to generate both cardiomyocytes and ECs theoretically might be advantageous for cardiac regeneration, which will likely require both blood vessel formation and cardiac muscle regeneration. hESC-derived Flk- $1^{+} \mathrm{PdgfR}-\alpha^{+}$and SSEA- $1^{+} \mathrm{CPC}$ have the potential to differentiate into cardiomyocytes and ECs in vitro $(6,33)$; however, whether these CPCs contribute to the formation of functional blood vessels in vivo remains to be further established. Additionally, SSEA- $1^{+}$CPCs appear to be at an early developmental stage, expressing both OCT4 and MESP1/2. Moreover, utilizing these surface receptors as markers for CPC isolation is not reliable, since the expression of Flk-1, PdgfR- $\alpha$, and SSEA-1 does not correlate well with cardiac differentiation $(7,34)$.

We extended the development of our experimental approach to generate highly committed and enriched hESC-derived ISL1-CPCs $\left(94.3 \pm 4.5 \%\right.$ ISL1 $\left.^{+} / \mathrm{NKX}_{2} .5^{+}\right)$. We observed that hISL1-CPCs effectively differentiated into cardiomyocytes and ECs and formed functional small blood vessels in vivo, providing a scientific foundation to consider heterologous ISL1-CPCs as a cell-based therapy for patients. Application of this strategy to acute MI would require approaches to address the immunogenicity of these heterologous cells, such as short-term blockage of leukocyte costimulatory molecules, which efficiently promotes engraftment of ESCs and their derivatives in an animal model (35). We could envision using hESC-derived ISL1-CPCs coupled with transient immune modulation. However, in patients with chronic ischemic cardiomyopathy, there would be time to generate autologous ISL1-CPCs for cardiac repair from patient-specific iPSCs, which mimic ESCs and can be derived from a patient's own somatic cells via expression of stem cell factors. This approach is advantageous in not requiring immune modulation. There are still many challenges ahead to optimize our approach. However, these initial results suggest that ISL1CPCs and the robust methylcellulose spheroid delivery approach should be further evaluated in preclinical cardiac repair studies in large animal models and ultimately might represent a therapeutic intervention in patients with ischemic cardiomyopathy and heart failure.

\section{Methods}

Isolation and culture of mISL1-CPCS. ISL1-CPCs were isolated based on GFP expression and FACS sorter and were plated on growth factor-reduced Matrigel-coated (1:30) 24-well plates after sorting or in suspension for 24 hours for spheroid formation. ISL1-CPC single cells were cultured for 7 days and ISL1-CPC spheroids for 6 days for differentiation. $10^{5}$ cells (ISL1-CPC single cells) or 100 spheroids (1,000 cells/ spheroid) were plated per well. Additional details are available in the Supplemental Materials.

hISL1-CPCs. hESC line H9 (from Joseph C. Wu, Stanford University, Stanford, California, USA) (36) containing pUb-fluc-mRFP-tTk for cell tracking was maintained in mTeSR1 medium on Matrigel-coated 
plates (1:30) to $85 \%-90 \%$ confluency. Modifications, including the use of glass chamber slide and harvesting hISL1-CPCs on day 6 , were made in the protocol originally described to derived cardiomyocytes from hESC or iPSCs (16). Additional details are available in the Supplemental Materials.

Methylcellulose-induced ISL1-CPC spheroid formation and treatments. ISL1-CPC spheroids were generated following the procedure described for ECs (18). Briefly, a suspension of ISL1-CPCs containing 10 cells/ $\mu 1$ was prepared in $0.24 \%$ methylcellulose-containing medium, and $100 \mu 1$ of cell suspension was added to each well (low attachment, round bottom 96-well plates) in the presence or absence of various small molecules. Spheroids were typically formed 12-24 hours after cell seeding. Additional details are available in the Supplemental Materials.

Experimental animals: surgeries, experimental treatments, and echocardiography. Female SCID-bg mice (Taconic Biosciences) were subjected to ligation of the left coronary artery at 9-12 weeks old. Immediately after coronary artery ligation, mISL1-CPC spheroids (500 spheroids/animal, 1,000 cells/spheroid) or hISL1-CPC spheroids (2,000 spheroids/animal, 1,000 cells/spheroid) were suspended in $20 \mu 1$ of growth factor-reduced Matrigel, followed by a single injection into the peri-infarct area using insulin syringes with a 45-degree angle 31-G needle. Echocardiography images were recorded using a Vevo 2100 System at 48 hours, 2 weeks, and 4 weeks after surgery. Additional details are available in the Supplemental Materials.

Bioluminescence imaging. D-Luciferin sodium salt $(1.5 \mathrm{mg})$ in DPBS was i.p. injected per $10 \mathrm{~g}$ of mouse body weight. Animals were anesthetized with $2 \%-2.5 \%$ isoflurane $/ \mathrm{O}_{2}(\mathrm{v} / \mathrm{v})$ in a chamber. Images were recorded 15 minutes after luciferin injection using an IVIS 200 (PerkinElmer) (mISL1-CPC) and IVIS Spectrum (PerkinElmer) (hISL1-CPC) with animals maintained at $1 \%-1.5 \%$ isoflurane $/ \mathrm{O}_{2}$ inhaled anesthesia and analyzed with Living Image 4.3.1. Background signals from control animals were subtracted from the signal individually recorded for animals injected with ISL1-CPC spheroids at the 3 time points described.

Statistics. Statistical analyses were performed with GraphPad Prism 6. Repeated measurements 1-way ANOVA with Geisser-Greenhouse correction was used to compare the bioluminescence of ISL1-CPCs at different time points after implantation. Two-way ANOVA was used to analyze the effect of different treatments (control or cell injection) at various time points after surgery. Bioluminescence data at different time points and echocardiographic comparisons 48 hours or 2-3 days versus 4 weeks, as well as data obtained with human cardiac fibroblasts, were analyzed with paired 2-tailed Student's $t$ test. For qPCR, results were analyzed with Mann-Whitney $U$ test. Group survival was analyzed with the Logrank Mantel-Cox test. Unpaired 2-tailed Student's $t$ test was used for the rest of the pair comparisons.

Study approval. Mouse and rat maintenance was performed following the American Association for Laboratory Animal Care guidelines. Surgical procedures including before and after operative care and treatments were approved by Yale's Institutional Animal Care and Use Committee (IACUC).

An extended version of Experimental Procedures is available in the Supplemental Materials.

\section{Author contributions}

$\mathrm{OB}$ and YQ contributed to the conceptualization of the study and design of the experimental procedures. ZWZ and YH performed surgeries. LHY provided technical advice for ultrasound imaging. NM and AB conducted ultrasound imaging and analysis. LW and DSK provided Y-chromosome FISH probe. WC and JSP provided advice for spheroid formation. OB performed all the experiments and statistical analyses. OB and YQ wrote the manuscript, and OB, CS, DSK, LHY, JSP, and YQ reviewed and edited the manuscript. $\mathrm{OB}$ and $\mathrm{YQ}$ obtained funding support for the study.

\section{Acknowledgments}

We thank Joseph C. Wu from Stanford University for sharing the pUb-fluc-mRFP-tTk lentiviral construct and hESC line H9 containing pUb-fluc-mRFP-tTk for cell tracking. This study was supported by Connecticut Government Stem Cell Seed Grant 13-SCA-YALE-32 (to O. Bartulos), AHA 09SDG2080420, NIH 1K02HL101990-01, CT Stem Cell 11SCB18, and CTSA UL1 RR024139 (to Y. Qyang), NIH R01 HL128069 (L.H. Young), and NIH R01-HL085416 (to J.S. Pober).

Address correspondence to: Yibing Qyang, 300 George Street, Room 773A, New Haven, Connecticut 06511, USA. Phone: 203.737.6354. E-mail: yibing.qyang@yale.edu. 
1. van Berlo JH, et al. c-Kit ${ }^{+}$cells minimally contribute cardiomyocytes to the heart. Nature. 2014;509(7500):337-341.

2. Murry CE, et al. Haematopoietic stem cells do not transdifferentiate into cardiac myocytes in myocardial infarcts. Nature. 2004;428(6983):664-668.

3. Rando TA. Stem cells, ageing and the quest for immortality. Nature. 2006;441(7097):1080-1086.

4. Smits AM, et al. Human cardiomyocyte progenitor cell transplantation preserves long-term function of the infarcted mouse myocardium. Cardiovasc Res. 2009;83(3):527-535.

5. Smith RR, et al. Regenerative potential of cardiosphere-derived cells expanded from percutaneous endomyocardial biopsy specimens. Circulation. 2007;115(7):896-908.

6. Fernandes $\mathrm{S}$, et al. Comparison of human embryonic stem cell-derived cardiomyocytes, cardiovascular progenitors, and bone marrow mononuclear cells for cardiac repair. Stem Cell Reports. 2015; 5(5): 753-762.

7. Kattman SJ, et al. Stage-specific optimization of activin/nodal and BMP signaling promotes cardiac differentiation of mouse and human pluripotent stem cell lines. Cell Stem Cell. 2011;8(2):228-240.

8. Menasche P, et al. Human embryonic stem cell-derived cardiac progenitors for severe heart failure treatment: first clinical case report. Eur Heart J. 2015;36(30):2011-2017

9. Cai CL, et al. Isl1 identifies a cardiac progenitor population that proliferates prior to differentiation and contributes a majority of cells to the heart. Dev Cell. 2003;5(6):877-889.

10. Ma Q, Zhou B, Pu WT. Reassessment of Is11 and Nkx2-5 cardiac fate maps using a Gata4-based reporter of Cre activity. Dev Biol. 2008;323(1):98-104.

11. Moretti A, et al. Multipotent embryonic is $11^{+}$progenitor cells lead to cardiac, smooth muscle, and endothelial cell diversification. Cell. 2006;127(6):1151-1165.

12. Bu L, et al. Human ISL1 heart progenitors generate diverse multipotent cardiovascular cell lineages. Nature. 2009;460(7251):113-117.

13. Qyang Y, et al. The renewal and differentiation of Is $11^{+}$cardiovascular progenitors are controlled by a Wnt/beta-catenin pathway. Cell Stem Cell. 2007;1(2):165-179.

14. Soh BS, et al. Endothelin-1 supports clonal derivation and expansion of cardiovascular progenitors derived from human embryonic stem cells. Nat Commun. 2016;7:10774.

15. Ahlgren U, Pfaff SL, Jessell TM, Edlund T, Edlund H. Independent requirement for ISL1 in formation of pancreatic mesenchyme and islet cells. Nature. 1997;385(6613):257-260.

16. Lian X, et al. Directed cardiomyocyte differentiation from human pluripotent stem cells by modulating Wnt/beta-catenin signaling under fully defined conditions. Nat Protoc. 2013;8(1):162-175.

17. Gago-Lopez N, et al. THY-1 receptor expression differentiates cardiosphere-derived cells with divergent cardiogenic differentiation potential. Stem Cell Reports. 2014;2(5):576-591.

18. Alajati A, et al. Spheroid-based engineering of a human vasculature in mice. Nat Methods. 2008;5(5):439-445.

19. Laflamme MA, et al. Cardiomyocytes derived from human embryonic stem cells in pro-survival factors enhance function of infarcted rat hearts. Nat Biotechnol. 2007;25(9):1015-1024.

20. Clement S, et al. Expression and function of alpha-smooth muscle actin during embryonic-stem-cell-derived cardiomyocyte differentiation. J Cell Sci. 2007;120(Pt 2):229-238.

21. Emami KH, et al. A small molecule inhibitor of beta-catenin/CREB-binding protein transcription [corrected]. Proc Natl Acad Sci U S A. 2004;101(34):12682-12687.

22. Sundberg TB, et al. Disruption of Wnt planar cell polarity signaling by aberrant accumulation of the MetAP-2 substrate Rab37. Chem Biol. 2011;18(10):1300-1311.

23. Burridge K, Wennerberg K. Rho and Rac take center stage. Cell. 2004;116(2):167-179.

24. Noritake J, Watanabe T, Sato K, Wang S, Kaibuchi K. IQGAP1: a key regulator of adhesion and migration. J Cell Sci. 2005;118(Pt 10):2085-2092.

25. Coso OA, Teramoto H, Simonds WF, Gutkind JS. Signaling from G protein-coupled receptors to c-Jun kinase involves beta gamma subunits of heterotrimeric G proteins acting on a Ras and Rac1-dependent pathway. J Biol Chem. 1996;271(8):39633966.

26. Lin Z, et al. Pi3kcb links Hippo-YAP and PI3K-AKT signaling pathways to promote cardiomyocyte proliferation and survival. Circ Res. 2015;116(1):35-45.

27. Haskins JW, Nguyen DX, Stern DF. Neuregulin 1-activated ERBB4 interacts with YAP to induce Hippo pathway target genes and promote cell migration. Sci Signal. 2014;7(355):ra116.

28. Li Z, et al. Imaging survival and function of transplanted cardiac resident stem cells. J Am Coll Cardiol. 2009;53(14):1229-1240.

29. Galindo CL, et al. Anti-remodeling and anti-fibrotic effects of the neuregulin-1beta glial growth factor 2 in a large animal model of heart failure. J Am Heart Assoc. 2014;3(5):e000773.

30. Chong JJ, et al. Human embryonic-stem-cell-derived cardiomyocytes regenerate non-human primate hearts. Nature. 2014;510(7504):273-277.

31. Ye L, et al. Cardiac repair in a porcine model of acute myocardial infarction with human induced pluripotent stem cell-derived cardiovascular cells. Cell Stem Cell. 2014;15(6):750-761.

32. Kawamura M, et al. Feasibility, safety, and therapeutic efficacy of human induced pluripotent stem cell-derived cardiomyocyte sheets in a porcine ischemic cardiomyopathy model. Circulation. 2012;126(11 Supp1 1):S29-S37.

33. Blin G, et al. A purified population of multipotent cardiovascular progenitors derived from primate pluripotent stem cells engrafts in postmyocardial infarcted nonhuman primates. J Clin Invest. 2010;120(4):1125-1139.

34. Mummery CL, Zhang J, Ng ES, Elliott DA, Elefanty AG, Kamp TJ. Differentiation of human embryonic stem cells and induced pluripotent stem cells to cardiomyocytes: a methods overview. Circ Res. 2012;111(3):344-358.

35. Pearl JI, et al. Short-term immunosuppression promotes engraftment of embryonic and induced pluripotent stem cells. Cell Stem Cell. 2011;8(3):309-317.

36. Wang Y, et al. Genome editing of human embryonic stem cells and induced pluripotent stem cells with zinc finger nucleases for cellular imaging. Circ Res. 2012;111(12):1494-1503. 